\title{
Single-cell transcriptomic analysis elucidates APOE genotype specific changes across cell types in two brain regions in Alzheimer's disease
}

\section{Stella Belonwu}

University of California San Francisco

\section{Yaqiao Li}

University of California, San Francisco

\section{Daniel Bunis}

University of California, San Francisco

\section{Arjun Arkal Rao}

University of California, San Francisco

\section{Caroline Warly Solsberg}

University of California, San Francisco

\section{Tomiko Oskotsky}

UCSF Bakar Computational Health Sciences Institute, University of California San Francisco, San

Francisco, CA, USA https://orcid.org/0000-0001-7393-5120

\section{Alice Taubes}

University of California, San Francisco

\section{Brian Grone}

Gladstone Institutes

\section{Kelly Zalocusky}

Gladstone Institutes

\section{Gabriela Fragiadakis}

University of California, San Francisco

\section{Yadong Huang}

Gladstone Institutes https://orcid.org/0000-0002-5871-4589

\section{Marina Sirota ( $\square$ Marina.Sirota@ucsf.edu )}

UCSF Bakar Computational Health Sciences Institute, University of California San Francisco, San

Francisco, CA, USA https://orcid.org/0000-0002-7246-6083

\section{Article}

Keywords: Alzheimer's Disease (AD), transcriptomic analysis, genomics 
Posted Date: March 15th, 2021

DOI: https://doi.org/10.21203/rs.3.rs-291648/v1

License: (c) (i) This work is licensed under a Creative Commons Attribution 4.0 International License. Read Full License 
Single-cell transcriptomic analysis elucidates $A P O E$ genotype specific changes across cell types in two brain regions in Alzheimer's disease.

Stella A. Belonwu ${ }^{1,2}$, Yaqiao $\mathrm{Li}^{1,2}$, Daniel Bunis ${ }^{1,3,4}$, Arjun Arkal Rao ${ }^{3,4,5}$, Caroline Warly Solsberg ${ }^{1,2}$, Tomiko Oskotsky ${ }^{1,6}$, Alice Taubes ${ }^{1,7}$, Brian Grone ${ }^{7}$, Kelly Zalocusky ${ }^{7}$, Gabriela K. Fragiadakis $^{3,4,9}$, Yadong Huang ${ }^{5,7,8}$, Marina Sirota ${ }^{1,6^{*}}$

\author{
Affiliations: \\ ${ }^{1}$ Bakar Computational Health Sciences Institute, University of California, San Francisco, San \\ Francisco, CA, USA \\ ${ }^{2}$ Pharmaceutical Sciences and Pharmacogenomics Graduate Program, University of \\ California, San Francisco, San Francisco CA, USA \\ ${ }^{3}$ CoLabs, University of California, San Francisco, San Francisco, CA, USA \\ ${ }^{4}$ Bakar ImmunoX Initiative, University of California, San Francisco, San Francisco, CA, USA \\ ${ }^{5}$ Department of Pathology, University of California, San Francisco, San Francisco, CA, USA \\ ${ }^{6}$ Department of Pediatrics, University of California, San Francisco, San Francisco, CA, USA \\ ${ }^{7}$ Gladstone Institute of Neurological Disease, San Francisco, CA, USA \\ ${ }^{8}$ Department of Neurology, University of California, San Francisco, San Francisco, CA, USA \\ ${ }^{9}$ Department of Medicine, Division of Rheumatology, University of California, San Francisco, \\ San Francisco, CA, USA
}

*Corresponding author: Marina Sirota (marina.sirota@ucsf.edu) 


\begin{abstract}
Alzheimer's Disease (AD) is a complex neurodegenerative disease that gravely affects patients and imposes an immense burden on caregivers. Apolipoprotein E4 (APOE4) has been identified as the most common genetic risk factor for $\mathrm{AD}$, yet the molecular mechanisms connecting APOE4 to AD are not well understood. Past transcriptomic analyses in $\mathrm{AD}$ have revealed $A P O E$ genotype-specific transcriptomic differences; however, these differences have not been explored at a single-cell level. Here, we leverage the first two single-nucleus RNA sequencing AD datasets from human brain samples, including nearly 55,000 cells from the prefrontal and entorhinal cortices. We observed more global transcriptomic changes in APOE4 positive AD cells and identified differences across $A P O E$ genotypes primarily in glial cell types. Our findings highlight the differential transcriptomic perturbations of APOE isoforms at a single-cell level in $\mathrm{AD}$ pathogenesis and have implications for precision medicine development in the diagnosis and treatment of $\mathrm{AD}$.
\end{abstract}


Alzheimer's disease (AD) is a heterogeneous neurodegenerative disorder, which accounts for at least $60 \%$ of dementia cases ${ }^{1}$. Further underscoring the importance of $\mathrm{AD}$ research, cases of $\mathrm{AD}$ are projected to increase by more than 3 -fold by 2050 , yet there currently are no disease altering treatments ${ }^{2,3}$. AD is defined by pathological hallmarks of aggregated extracellular amyloid- $\beta$ (A $\beta$ ) plaques, and intracellular tau neurofibrillary tangles ${ }^{1,4}$. As a complex disease, $A D$ has a number of environmental risk factors. Demographic risk factors include advanced age, low education level, and female sex. $\mathrm{AD}$ genetic risk factors such as $\mathrm{A} \beta$ precursor protein $(A P P)$, presenilin 1 (PSEN1), and presenilin 2 (PSEN2) lead to dominantly inherited early-onset AD and account for $<1 \%$ of $\mathrm{AD} \operatorname{cases}^{1,4,5}$.

The strongest genetic risk factor for late-onset or sporadic $\mathrm{AD}$ is the $\varepsilon 4$ allele of the apolipoprotein $\mathrm{E}(A P O E)$ gene. In humans, there are three common $A P O E$ allelic variants $(\varepsilon 2$, $\varepsilon 3$, and $\varepsilon 4$ ), which differ based on single substitutions at amino acid residues 112 and 158, with the most common $\varepsilon 3$ allele generally considered as a neutral form ${ }^{4,6,7}$. While the $\varepsilon 2$ allele is considered protective, the $\varepsilon 4$ allele is associated with increasing the risk of developing $\mathrm{AD}$ in a gene dose dependent manner ${ }^{4,8}$. Specifically, one copy of the $\varepsilon 4$ allele of $A P O E$ increases the risk of developing AD by 3 - to 4 -fold, and two copies increases this risk by 12 - to 15 -fold ${ }^{4,5}$.

APOE is a lipid-binding protein which serves a central role in regulating lipid transport and metabolism. It is highly expressed in the liver and brain, where it is primarily expressed in astrocytes ${ }^{4,6}$. APOE's functionality in the central nervous system has implications for AD in both $\mathrm{A} \beta$-dependent and $\mathrm{A} \beta$-independent pathways. For instance, in addition to regulating $\mathrm{A} \beta$ clearance, APOE regulates lipoprotein metabolism, supports cell proliferation, repairs 
membranes, supports myelination, and maintains blood brain barrier (BBB) integrity ${ }^{4,6,9}$. In regard to APOE isoforms, APOE4 has been linked to promoting $\mathrm{A} \beta$ retention by blocking its LRP1-mediated clearance ${ }^{9,10}$, insulin resistance through impaired insulin signaling ${ }^{11}, \mathrm{BBB}$ dysfunction and increased permeability ${ }^{8,12}$, and regulating glycogen synthase kinase 3 (GSK3), a kinase highly involved in phosphorylation of tau ${ }^{6,13}$. Our study aims to identify transcriptomic differences associated with APOE isoforms at a single-cell level to better understand the underlying mechanisms contributing to AD pathophysiology and their specificity to each isoform. Transcriptomics represent a valuable means of understanding molecular underpinnings in disease conditions ${ }^{10,14-18}$; however, to our knowledge, in AD, APOE isoforms are yet to be investigated at a single-cell level, which can depict molecular profiles that would be otherwise masked in a bulk analysis.

In recent years, single-cell transcriptomic datasets were generated from the prefrontal ${ }^{19}$ and entorhinal ${ }^{20}$ cortices of human AD patients. With single-nucleus transcriptomes from the prefrontal cortex, Mathys and colleagues performed cell type specific differential expression analyses using 80,660 droplet-based nuclei across 24 individuals with varying degrees of AD pathology and 24 non-AD individuals, across six major cell types: excitatory neurons, inhibitory neurons, astrocytes, oligodendrocytes, oligodendrocyte precursor cells (OPCs), and microglia ${ }^{19}$. On examining $A P O E$ expression, they report that $A P O E$ was strongly upregulated in $\mathrm{AD}$-specific microglia, but downregulated in astrocytes in $\mathrm{AD}$; however, the authors did not look at the effects of $A P O E$ genotype. With single-nucleus transcriptomes from the entorhinal cortex, Grubman and colleagues analyzed 13,214 droplet-based nuclei from 6 men and women with AD and 6 non-AD sex and age matched controls ${ }^{20}$. Similar to Mathys et al., neurons, astrocytes, 
oligodendrocytes, OPCs, and microglia were surveyed. APOE expression was upregulated in an AD-specific microglial subpopulation and downregulated in AD-specific OPC and astrocyte subpopulations, which is consistent with findings from Mathys et al. Again, similar to Mathys et al., the relationship between $A P O E$ genotype and gene expression was not examined.

To expand on these single-cell analyses, we explore the cell type specific transcriptomic effects of $A P O E$ genotype across two brain regions: the prefrontal and entorhinal cortices, using these two publicly available datasets. In this study, we aim to answer the following questions: 1) Which cell types are most affected at the transcriptomic level by $A P O E$ genotype in the context of AD? 2) What are the global and cell type specific transcriptomic changes with respect to $A P O E$ genotype in the context of $\mathrm{AD}$ ? and 3) Are there any transcriptomic changes that are specific to APOE4 that better explain AD pathophysiology?

\section{Results}

\section{Sample classification and analytic workflow}

We classified samples based on tau tangle and $A \beta$ plaque burdens, using Braak clinical staging and Consortium to Establish a Registry for Alzheimer's Disease (CERAD) scores, respectively (AD: Braak stage $\geq$ IV, CERAD score $\leq 2$; Control: Braak stage I-III, CERAD score $\geq 3$ ) (Fig. 1). Following, from the prefrontal cortex cohort (Table 1), we analyzed single nucleus RNA-Seq (snRNA-seq) data containing 43,831 cells (Supplementary Table 1) and 17,593 genes, and from the entorhinal cortex cohort (Table 2), we analyzed snRNA-seq data containing 9,587 cells (Supplementary Table 2) and 10,850 genes. Both datasets were acquired from different sets of individuals. To examine cell type specific differences in gene expression in APOE3/3 
(homozygous for allele $\varepsilon 3$ ) and APOE3/4 (heterozygous $\varepsilon 3 / \varepsilon 4$ ) cells, we performed an APOE genotype stratified differential gene expression (DGE) analysis comparing age-matched AD cases to controls, with sex as a covariate, in excitatory (Ex), and inhibitory (In) neurons for the prefrontal cortex specifically, undistinguished neurons (Neu) for the entorhinal cortex, and astrocytes (Ast), microglia (Mic), oligodendrocytes (Oli), and oligodendrocyte progenitor cells (OPCs) for both cohorts (Supplementary Fig. 1). Differentially expressed genes (DEGs) were selected using cutoffs of a Benjamini-Hochberg (BH) adjusted p-value $<0.05$ and $\log 2$ fold change $>0.25$. DEGs were further passed as inputs to identify pathways for subsequent network analysis. We examined gene expression and network changes in $\mathrm{AD}$ compared to non- $\mathrm{AD}$ samples to identify cell type specific and universal changes based on APOE genotype (Fig. 1). Due to sample limitations for relatively rare $A P O E$ genotypes, we focused our analysis on comparisons between $\mathrm{AD}$ and non- $\mathrm{AD}$ groups with an $\mathrm{APOE} 3 / 3$ or $\mathrm{APOE} 3 / 4$ genotype in this study.

\section{DGE analysis in the prefrontal cortex identifies distinct AD-related changes in astrocytes, oligodendrocytes, and OPCs in APOE3/4 versus APOE3/3 cell type specific disease signatures}

Leveraging data from Mathys et al, we identified DEGs in all cell type and APOE genotype pairings when comparing $\mathrm{AD}$ to non-AD samples from 43,831 cells covering 17,593 genes. Interestingly, DEGs were primarily downregulated in APOE3/4 astrocytes, oligodendrocytes and OPCs, while they were primarily upregulated in both APOE3/3 and APOE3/4 neurons (Fig. 2a). Altogether, across all cell types we identified 278 unique DEGs (Supplementary Table 3). Of the 278 DEGs, 8 were specific to APOE3/3 and 135 were specific to APOE3/4. We observed DEGs 
previously linked to $\mathrm{AD}\left(C L U^{5,21}, C C K^{22-24}, N R G N^{25,26}, D H F R^{27,28}, E R B B 4^{29,30}, N R X N 1^{31}\right)$, which were shared by APOE3/3 and APOE3/4 cells. In most cases, expression differences in these genes were in the same direction across genotypes, but with greater fold changes in APOE3/4 as compared to APOE3/3 cells (Fig. 2b). Across cell types, while the majority of DEGs were shared and in consistent direction across APOE3/3 and APOE3/4 cells (Fig. 2c, yellow color; Supplementary Fig. 2), there were a few shared DEGs with opposite directionality of expression changes, such as DOCK4 in microglia, SPARCL1 in neurons, and FRYL in oligodendrocytes (Fig. 2c, pink color; Supplementary Fig. 2).

Notably, some DEGs in AD patients relative to controls were shared across multiple cell types (Fig. 3a). For example, APP binding family B member 1 interacting protein $(A P B B 1 I P)$, and $D O C K 8$, a protein highly involved in brain development and immune response ${ }^{32}$, were differentially expressed in most APOE3/4 cell types and in APOE3/3 neurons. Interestingly, for both $A P B B 1 I P$ and $D O C K 8$, we observed cell-type specific effects. Both genes were downregulated in astrocytes, oligodendrocytes and OPCs and upregulated in microglia and neurons from APOE3/4 AD patients versus APOE3/4 controls. In APOE3/3 individuals, both genes were only significantly upregulated in neurons in AD patients versus controls. APOE itself was also differentially expressed in AD patients versus controls, with an increase in both APOE3/4 and APOE3/3 neurons and in APOE3/3 microglia as well as a decrease in APOE3/4 astrocytes, oligodendrocytes, and OPCs. MTRNR2L12 expression, which encodes a humanin isoform necessary for neuroprotection and anti-apoptotic function suggested to have utility as a blood marker for cognitive disability and early dementia for adults with Down Syndrome ${ }^{33,34}$, was very similar to APOE expression. Hierarchical clustering of samples using AD compared to 
control pseudobulk cell type gene expression (Fig. 2d) showed clustering of samples dominated by $A P O E$ genotype before cell type identity for all cell types except neurons, which instead cluster by cell type. Generally, we observed more similarities in DEGs of AD versus control across $A P O E$ genotypes in neuronal populations (both excitatory and inhibitory neurons), and differences primarily in non-neuronal cells (astrocytes, oligodendrocytes, and OPCs). In addition to identifying shared DEGs across cell types and $A P O E$ genotypes, we also observed a larger range of $\log 2$ fold change in the analysis of APOE3/4 AD versus control $(-0.834,1.032$; median $=-0.273)$ compared to the analysis of $\mathrm{APOE} 3 / 3 \mathrm{AD}$ versus control $(-0.503,1.115$; median $=$ 0.342), which we visualized in a number of shared DEGs such as LINGO1, NRXN1, RASGEF1B, and $C L U$ (Fig. 3b).

As the prefrontal cohort contained a sole non-AD sample with the APOE3/4 genotype from a male donor, we performed a sensitivity analysis in cells from males to determine whether the same gene signatures from comparing $\mathrm{AD}$ to control tissue are present. We identified 300 unique DEGs across all cell types (Supplementary Fig. 3; Supplementary Table 4). Of these DEGs, 18 were specific to APOE3/3 cells and 128 to APOE3/4 cells. Similar to the previous analysis, we observed more differences in perturbed gene profiles across $A P O E$ genotypes in astrocytes, oligodendrocytes, and OPCs, where DEGs were primarily downregulated in APOE3/4 cells. Additionally, clustering of DEGs by $\log 2$ fold change also showed a stronger clustering by APOE genotype than cell type identity (Supplementary Fig. 3). 


\section{DGE analysis in the entorhinal cortex identifies distinct AD-related changes in microglia and oligodendrocytes in APOE3/4 versus APOE3/3 cell type specific disease signatures}

Leveraging data from Grubman et al., we identified DEGs in all cell type and APOE genotype pairings when comparing AD to control tissue from 9,587 cells and 10,850 genes, where DEGs were primarily downregulated in APOE3/3 AD versus control and upregulated in APOE3/4 AD versus control (Fig. 4a). Altogether, across all cell types we identified 232 unique DEGs (Supplementary Table 5). Of the DEGs, 29 were specific to the APOE3/4 AD, and none were specific to the APOE3/3 AD. In each cell type, we observed more DEGs in the APOE3/4 comparison, some of which were shared with APOE3/3 analysis, though often with consistent opposite directionality (Fig. 4b; yellow (same) and pink (opposite) colors; Supplementary Fig. 2). We observed a higher proportion of common DEGs across $A P O E$ groups in microglia and oligodendrocytes than in other cell types, and in most cases, there was opposite directionality of gene expression changes between APOE3/3 AD versus control comparison and APOE3/4 AD versus control comparison. Overall, clustering samples using $\mathrm{AD}$ compared to control pseudobulk cell type gene expression (Fig. 4c) showed consistent clustering of samples by APOE genotype.

When surveying DEG overlaps across cell types in the entorhinal cortex, consistent with the prefrontal cortex analysis, we observed more DEGs overall shared across APOE3/4 cell types than across APOE3/3 cell types in AD (Supplementary Fig. 4). In the APOE3/3 case control comparisons, six DEGs $-A T P I B 1^{36}$, a sodium and potassium ATPase necessary for regulating ionic gradients; $C S T 3^{37}$, an AD risk factor; $G P C 5^{38}$, $a$ neurotrophic factor; $M E G 3^{39}$, a long noncoding RNA and apoptosis regulator; NRXN1; and LINC00486, a relatively uncharacterized long 
non-coding transcript - were shared by all cell types. LINC00486 was upregulated in all APOE3/3 cell types in AD, ATP1B1, GPC5, MEG3, and NRXN1 were downregulated in all APOE3/3 cell types in AD, and CST3 was downregulated in all APOE3/3 cell types in AD, except OPCs where it was upregulated. These DEG's were also reflected in APOE3/4 cells, with LINC00486 upregulated in all cell types, ATP1B1 and MEG3 upregulated in non-neuronal cell types, NRXN1 upregulated in oligodendrocytes and downregulated in all other cell types, GPC5 downregulated in astrocytes and upregulated in all other cell types, and CST3 downregulated in astrocytes and upregulated in neurons and oligodendrocytes in case control comparisons.

Overall, in the APOE3/4 case control comparisons, 87 DEGs were shared in all cell types, with 64 consistently upregulated in AD tissue and 23 with mixed directionality across cell types when comparing AD to control tissue (Supplementary Fig. 4). Of these shared DEGs, a few with higher absolute $\log 2$ fold changes between $\mathrm{AD}$ and controls include $M B P$, a gene important for myelination $^{40,41}$ that was upregulated in all APOE3/4 cell types in AD except oligodendrocytes, and LINGO1, which was upregulated in all APOE3/4 cell types as well as APOE3/3 astrocytes and OPCs in AD. Interestingly the average $\log 2$ fold change for LINGO1 in APOE3/4 AD samples (3.52) was much higher than that of the APOE3/3 AD samples (0.451). Additionally, protein folding $H S P A 1 A$, the neuroprotective chaperone and apoptosis regulator $C R Y A B^{42}$, and quinoid dihydropteridine reductase $(Q D P R)$ were upregulated in all APOE3/4 cell types in AD. However, HSPA1A was also downregulated in APOE3/3 microglia, oligodendrocytes, and OPCs, $C R Y A B$ was downregulated in APOE3/3 oligodendrocytes, and $Q D P R$ was downregulated in APOE3/3 microglia in AD. The latter two genes have previously been observed to be upregulated in oligodendrocytes and OPCs of pathologically confirmed AD individuals ${ }^{19}$, most 
of them are usually APOE4 carriers. We also observed a larger range of log 2 fold change in APOE3/4 cells $(-2.918,3.839$; median $=0.688)$ in AD compared to APOE3/3 cells $(-2.385$, 2.227; median $=-0.436$ ) in $\mathrm{AD}$, which we visualized in a number of shared DEGs such as LINGO1, NRXN1,FTL, and ADGRL3 (Supplementary Fig. 4). Largely, when comparing AD to non-AD cells in the entorhinal cortex, while we observed changes relevant to AD pathophysiology across APOE3/3 and APOE3/4 genotypes, we observed a number of flipped expression profiles across both $A P O E$ genotypes primarily in non-neuronal cells, and more universal transcriptional changes and changes of higher amplitude in APOE3/4 AD versus control comparison as compared to APOE3/3 AD versus control comparison.

\section{Comparative analysis across brain regions shows more AD-related transcriptomic changes in the entorhinal cortex compared to the prefrontal cortex, with consistent $A P O E$ genotype specific disease signatures}

We observed a higher number of DEGs and larger log2 fold change magnitudes across cell types in the entorhinal cortex than in the prefrontal cortex in AD. The number of shared DEGs within cell types across $A P O E$ genotype groups was highest in the entorhinal cortex in AD, while the number of shared DEGs within cell types across brain regions was highest in APOE3/4 cells in AD (Fig. 5a). With hierarchical clustering of per-cell and genotype group pseudobulk expression, we observed strongest clustering by brain region, followed by $A P O E$ genotype (Fig. $5 b)$. 


\section{Pathway and network analysis reveal $A P O E$ genotype specific perturbed biological processes primarily in glial cells across brain regions}

Pathway enrichment was performed using gprofiler ${ }^{43}$, a web server for functional enrichment of gene lists. Separate lists of upregulated and downregulated DEGs in AD relative to control, with a BH corrected adjusted p-value $<0.05$ and a relaxed absolute $\log 2$ fold change cutoff of above 0.1, in each cell type and $A P O E$ genotype were used as inputs for gprofiler (Supplementary Tables 6-9). A network analysis was performed to cluster the disease enriched pathways into biologically relevant groups using pathways with an adjusted $\mathrm{p}$-value $<0.01$ as inputs. Modules of biological themes were generated for each cell type based on the APOE genotype (Fig. 6; Supplementary Figures 5 and 6).

In astrocytes from the prefrontal cortex, we identified six enriched functional modules in both APOE3/3 and APOE3/4 AD relative to controls (Fig. 6a). We found five of these common modules (glutamatergic NMDA receptor transmission of glutamate; NLGN1 and NRXN1 clustering of NMDA receptor, periphery intrinsic plasma membrane function, trans synaptic anterograde signaling, transporter transmembrane activity) to be downregulated in $\mathrm{AD}$, and one, the LINGOI-TROY-NgR complex, which was previously suggested to be important for modulating glial-neuronal interactions in demyelinating lesions, upregulated in $\mathrm{AD}^{44}$. In APOE3/3 astrocytes, Ion and acid transport (receptor ion channel activity, regulator ion channel activity, acid sodium organic symporter, ferrous ferric ion binding, intracellular ferritin iron sequestering), glutamate receptor activity (mGLUR2, mGLUR3, mGLUR4, mGLUR7, mGLUR8), metabolic (aspartate uptake, astrocytic metabolism) as well as autolysosome activities (scavenging class receptors, secondary lysosome, autolysosome) were downregulated 
in AD, and myelin maintenance (PRNP, ASAH1), cell adhesion (FLRT3, LPHN3, UNC5B, UNC5D), and Vascular endothelial growth factor (VEGF) induced heat shock protein 90 (hsp90) complex were upregulated in $\mathrm{AD}$, indicating perturbation in processes important for autophagy and stress response which are known to accompany disease progression ${ }^{4,5}$. APOE3/4 astrocytes uniquely showed upregulation in pathways related to post-synaptic scaffold proteins (e.g., DLGAP1, DLG4, DLC1 and SHANK3) and actin assembly at cell junctions, but downregulation of synaptic membrane and neurotransmitter pathways, neurogenesis and nervous system development in AD.

In the entorhinal cortex, common astrocyte modules for $\mathrm{AD}$ cells relative to controls showed perturbation for processes such as aspartate update metabolism, astrocytic affinity, synaptic membrane organization, macromolecular cellular localization and transmembrane transport (Supplementary Fig. 6). In APOE3/3 astrocytes of the entorhinal cortex, we observed a downregulation of ion and neurotransmitter transport related pathways (intracellular ion and ferritin iron sequestering) in AD. APOE3/4 astrocytes in the entorhinal cortex had mostly upregulated pathway enrichment modules in $\mathrm{AD}$, in contrast to what was observed in prefrontal cortex. Many of these pathways governing cellular homeostasis, such as ATP synthesis, transmembrane cation transport, amyloid fibril formation and exosome regulation, and macromolecule and protein plasma membrane localization.

Microglia, the resident brain macrophage, contributes to neuroinflammation in AD and produces APOE upon activation in the brain ${ }^{1,4}$. Differentially enriched pathways were predominantly upregulated in APOE3/4 microglia in AD patients in both prefrontal (Supplementary Fig. 5) and 
entorhinal cortices (Fig. 6b). In APOE3/3 microglia, however, most significantly enriched pathways were downregulated in AD. Within the entorhinal cortex, changes in gliogenesis, myelination, cation transmembrane transport, cellular projection, synaptic spine development, and synaptic junction assembly pathway network modules were shared in APOE3/3 AD and APOE3/4 AD but perturbed in opposite directions, downregulated in APOE3/3 and upregulated in APOE3/4 microglia (Fig. 6b). The ITGAV-ITGB-SPPI complex, not previously linked to AD to our knowledge, was significantly upregulated in both brain regions in APOE3/3 microglia in $\mathrm{AD}$, but only in the prefrontal cortex in APOE3/4 microglia in AD (Fig. 6b and Supplementary Fig. 5). The downregulation of iron homeostasis and ferritin complex, a protein that binds to iron and reflects the level of iron storage in the body, was observed in APOE3/3 microglia and astrocytes of both prefrontal and entorhinal cortex in AD (Fig. 6a, Fig. 6b, and Supplementary Fig. 5).

Overall, network analysis comparing neurons from two brain regions yielded many similar perturbed biological processes within each APOE genotype in AD (Fig. 6c). In APOE3/3 neurons, shared differentially perturbed processes between brain regions were mostly related to regulation of membrane homeostasis (intrinsic integral membrane component, transporter transmembrane activity regulation), neuron projection (positive neurogenesis and differentiation regulation) and synaptic development (presynapse organization assembly, synapse assembly structure regulation, postsynaptic specialization assembly density). Pathway networks in APOE3/3 neurons specific to the prefrontal cortex pertain to cell structure development (actomyosin actin-based structure, extension growth development, anchoring junction, adherens cell), while the entorhinal cortex showed unique modules relevant to cellular energy production 
(oxidative respirasome synthesis, metabolic ATP nucleotide process). From APOE3/4 neurons, we observed a more diverse population of shared network modules between the two brain regions, including functional processes related to protein trafficking vesicles, myelination, membrane assembly, and voltage gated channel and neurotransmitter receptor regulation. Amyloid fibril formation was uniquely differentially regulated in APOE3/4 neurons and observed in both brain regions in $\mathrm{AD}$, while an amyloid beta precursor formation module was only observed APOE3/4 neurons in prefrontal cortex in AD.

In oligodendrocytes, which provide myelination, we observed common upregulation in proteasomal degradation (e.g., PSMA1) and the LINGO1-TROY-NgR complex, and downregulation in regulating ion activity in the prefrontal cortex (Supplementary Fig. 5) across $A P O E$ genotypes in AD. APOE3/3 oligodendrocytes in the prefrontal cortex showed an upregulation of the ITGAV-ITGB-SPPI complex and downregulation of pathways related to myelin organization (e.g., juxtaparanode region of axon) in AD. APOE3/4 oligodendrocytes, on the other hand, showed upregulation of stress responses through chaperone mediated protein folding, and downregulation of axon guidance and nervous system development processes in AD. In the entorhinal cortex, we observed modules of processes including neurogenesis, gliogenesis, amyloidosis, aerobic metabolic processes, and exocytosis to be upregulated in APOE3/4 cells and downregulated in APOE3/3 cells in AD (Supplementary Fig. 6). As in prefrontal cortex, for APOE3/3 oligodendrocytes, we found an upregulation for the ITGAVITGB-SPP1 complex and a downregulation for ion transport activity, protein refolding, and regulation of MAP kinase signaling activity (e.g., positive regulation of Erk1 and Erk2) in AD. 
Lastly, in APOE3/4 oligodendrocytes, we observed postsynaptic structural specialization to be uniquely downregulated

For OPCs in the prefrontal cortex, there were no common network modules across APOE genotypes. In APOE3/3 AD, we identified downregulation for brain cell development processes (AHI1-NPHP1-HAP1) (Supplementary Fig. 5). In APOE3/4 OPCs, we observed upregulated modules for the ferritin, GAIT and LINGOI-TROY-NgR complexes, and downregulation for glutamatergic synaptic activity, plasma membrane and cell organization, and lipoprotein density in $\mathrm{AD}$, which may have implications for neuronal integrity and lipid transport and metabolism. In the entorhinal cortex of $\mathrm{AD}, \mathrm{OPCs}$ across $\mathrm{APOE} 3 / 3$ and $\mathrm{APOE} 3 / 4$ genotypes shared modules for cell motility, neurogenesis and ion regulation synapse assembly, and neurotransmitter transport (Supplementary Fig. 6). We also observed upregulation of the LINGOI-TROY-NgR, and downregulation of glutamatergic signaling in APOE3/3 OPCs in AD. Specific to APOE3/4 OPCs in $\mathrm{AD}$, we identified upregulation of processes related to aerobic metabolic processes, stress response, autophagy, amyloid fibril regulation, demyelination, and immune response.

\section{Discussion}

As APOE4 is the greatest known genetic risk factor for $\mathrm{AD}$, we analyzed recently available single-cell transcriptomic datasets from two brain regions to better understand how APOE genotype plays into transcriptional profiles of $\mathrm{AD}$ in a cell type specific manner. We aimed to understand whether transcriptional differences exist, and if so, how they might be represented in different cell types across brain regions; which cell types were most affected by APOE genotype; what changes were shared or dissimilar across cell types; and whether such findings are 
consistent across brain regions. Our differential gene expression analysis involved a comparison between $\mathrm{AD}$ and control within each cell type, stratified by APOE genotype. Due to the limitations of the datasets, we restricted analysis to compare $\mathrm{AD}$ patients versus control individuals with an APOE3/3 or APOE3/4 genotype. In both the prefrontal and entorhinal cortices, we observed shared and unique gene signatures across these APOE genotypes that were often cell type specific, but sometimes spanned many cell types (Fig. 2, Fig. 3, Fig. 4, and Supplementary Fig. 4). Additionally, we observed more DEGs unique to APOE3/4 cells in AD versus control when compared to DEGs for APOE3/3 cells in AD versus control and more DEG overlaps across cell types in $\mathrm{APOE} 3 / 4 \mathrm{AD}$, suggesting a stronger and more global AD-related molecular response when one copy of the APOE4 allele is present.

In the prefrontal cortex, most DEGs that are common across cell types tend to be more strongly differentially expressed in APOE3/4 AD as compared to those in APOE3/3 AD. Additionally, we observed most of the APOE genotype specific changes in APOE3/4 astrocytes, oligodendrocytes and OPCs, where these genes are predominantly downregulated in $\mathrm{AD}$ as compared to controls. Neurons, on the other hand, tended to exhibit DEGs of AD versus control that were common across $A P O E$ genotypes (Fig. 2a and Supplementary Fig. 2). Through hierarchical clustering of samples using AD compared to control pseudobulk cell type gene expression (Fig. 2c), we observed clustering by APOE genotype in all cell types except neurons.

In the entorhinal cortex, microglia and oligodendrocytes had the highest proportion of DEGs of $A D$ versus control that were shared across $A P O E$ genotypes. Interestingly, these DEGs frequently exhibited opposite log fold-change direction between APOE3/3 AD cells and 
APOE3/4 AD cells. Additionally, through hierarchical clustering of samples using AD compared to control pseudobulk cell type gene expression, we observed that samples clustered primarily by $A P O E$ genotype (Fig. 4c). Compared to the prefrontal cortex, the entorhinal cortex, which is implicated in early stages of AD where tau begins to accumulate and the occurrence of synaptic and neuronal loss is associated with the onset of cognitive impairment ${ }^{1,4,45}$, had a larger number of DEGs of $\mathrm{AD}$ versus control in each cell type and larger log2 fold change range, implying a greater magnitude of molecular changes in this region in $\mathrm{AD}$, as compared to prefrontal cortex.

Through pathway and network analysis, we identified biological processes potentially involved in AD pathogenesis that were uniquely modified by APOE genotype (Fig. 6, Supplementary Fig. 5, and Supplementary Fig. 6). While many essential cellular processes were differentially regulated in $\mathrm{APOE} 3 / 3$ neurons in $\mathrm{AD}$, most were related to energy production, membrane regulation, and cellular signaling through synapse. APOE3/4 neurons in AD, on the other hand, demonstrated a perturbation of enriched pathways linked to myelination and protein trafficking vesicle regulation (both endocytosis and exosome), which are important cellular processes that protect the integrity of neurons by providing insulation and filtering toxic elements from these cells. This evidence suggests that APOE, a known lipid metabolizing protein, may play differential roles in maintaining essential metabolic processes for neuronal myelination and vesicle trafficking based on its isoform. Glial cells from APOE3/3 and APOE3/4 AD had many uniquely versus common altered biological processes, identified by the $A P O E$ genotype specific pathway modules. This suggests that $A P O E$ genotype modifies glial cell biology in different ways compared to its effects on neuronal cell biology during AD progression. Further study on 
AD pathogenesis focusing on glial cell modification by the APOE genotype might facilitate personalized therapeutic development for AD patients with different $A P O E$ genotypes.

While we were able to examine $A P O E$ genotype specific changes across cell types in both brain regions, some limitations exist. For one, as we did not have any APOE $4 / 4$ controls, we designed our analysis to examine only APOE3/3 and APOE3/4 samples. Each dataset contained only one APOE3/4 control, which was a male sample in both cases. We performed a sensitivity analysis in males of the prefrontal cortex cohort (Supplementary Fig. 3), where we also observed more differences in perturbed gene profiles across $A P O E$ genotypes in astrocytes, oligodendrocytes, and OPCs, and a stronger clustering by $A P O E$ genotype than cell type identity. We also observed an imbalance in the entorhinal cohort, where all female samples were APOE3/3 cells, and male APOE3/3 cases were not present. However, here we were able to mitigate this caveat by including sex as a covariate in our model for differential expression to account for the confounding variables introduced by the experimental design.

Secondly, due to the design of the Grubman et al. study, we observed a batch effect, where cases were sequenced in separate batches from controls, and each batch contained only one sex. To mitigate this limitation, we used Seurat's integration workflow and dimensionality reduction to confirm appropriate batch correction and included sex as a covariate in our model (Supplementary Fig. 1). Comparing across brain regions, we recognize that reasons for the differences we observed include the variability in acquiring each cohort, which is sourced from different sets of individuals and studies. The expected variability inherent in human studies also raises the concern that a limited number of single nucleus transcriptomes $(\mathrm{n}=30$ for the 
prefrontal cortex, $\mathrm{n}=9$ for the entorhinal cortex) may not be reflective of the greater human population. Although this concern is mitigated by the consistent observation that variance in gene expression is explained more by APOE genotype than cell type specificity, it underscores the need to extend these findings to a larger and more diverse population of human subjects in the future. The nature of our analysis only allows for association of transcriptomic changes with APOE genotype, so links to causality might be hypothesized, but additional followup are needed to prove any such potential links.

Moreover, we hope that future studies will generate more data from diverse sets of individuals, across different ages, racial and ethnic backgrounds, with a wider variety of APOE genotypes, and more brain regions, thus allowing for even more extensive insights. With more diverse genomic data, researchers may be able to 1) examine $\mathrm{AD}$ relative to control expression differences based on the number of copies of the $\mathrm{AD}$ risk variant, 2) stratify by age groups to determine whether changes are age-dependent, and 3) identify more concrete changes that are unique to and shared by different regions of the brain. Ultimately, we identified key AD-related genes and pathways that are specific to $A P O E$ genotypes and cell types, especially glial cells, as well as certain consistently affected pathways. These results will inform how glial cells are potentially primary sites of AD-related transcriptional differences based on APOE genotype, suggesting possible mechanisms and vulnerable cell subpopulations relevant to $\mathrm{AD}$ pathogenesis, and thus can help to facilitate precision medicine diagnostic and drug discovery efforts. 


\section{Methods}

\section{Materials Availability}

This study did not generate new unique reagents.

\section{Data and Code Availability}

Single nuclei RNA-Seq data and metadata were accessed from their respective repositories: the prefrontal cortex from the Accelerating Medicines Partnership Alzheimer's Disease Project (AMP-AD) Knowledge Portal under the Religious Orders Study and Memory and Aging Project (ROSMAP) (https://www.synapse.org/\#!Synapse:syn18485175;

https://www.synapse.org/\#!Synapse:syn3157322), and the entorhinal cortex from a data repository provided by Grubman et al. (http://adsn.ddnetbio.com/). Data from the entorhinal cortex are also available from the Gene Expression Omnibus (GEO) under the accession number GSE138852. To enable other researchers to explore these datasets, all code necessary for recreating the reported analyses and figures within $\mathrm{R}$, are available on Github at https://github.com/stebel5/AD_APOE_snRNAseq.

\section{Study Cohort Identification}

We acquired publicly available single nuclei RNA datasets from repositories specified by the first two single-cell transcriptomic AD studies ${ }^{19,20}$. Samples were classified based on tau neurofibrillary tangles, and amyloid $\beta(\mathrm{A} \beta)$ plaque burden, using Braak clinical staging and Consortium to Establish a Registry for Alzheimer's Disease (CERAD) scores. Cases were identified as individuals with severe tau deposition (Braak stage $\geq I V$ ), and high A $\beta$ load (CERAD score $\leq 2$ ), while non-AD Controls were identified as individuals with low tau (Braak 
stage I-III) and low A $\beta$ load (CERAD score $\geq 3$ ). The prefrontal cortex dataset consisted of age and sex matched samples from 48 individuals with varying degrees of AD pathology. For $A P O E$ genotype stratified analysis, we focused on APOE3/3 and APOE3/4, which consisted of 14 APOE3/3 controls, 1 APOE3/4 control, 9 APOE3/3 cases and 8 APOE3/4 cases (Table 1). The entorhinal cortex dataset initially consisted of age and sex matched samples from 6 AD and 6 control subjects, which were classified based on pathological analysis of amyloid $\beta$ plaques, Braak clinical staging, and cognitive impairment records from corresponding treating general practitioners. All cases in this cohort have numerous diffuse and neuritic amyloid beta plaques, and a Braak staging score of VI. For our APOE-stratified analysis, we focused on APOE3/3 and APOE3/4 cells, which restricted the dataset to 4 cases, and 5 controls (Table 2). Three of the cases were from APOE3/4 individuals, while one was from an APOE3/3 individual, and of the controls, four were from APOE3/3 individuals and the one was from an APOE3/4 individual.

\section{Single Cell Data Processing, Cell Type Identification and Batch Correction}

All data processing was conducted separately for each dataset with $\mathrm{R}^{46}$ version 4.0.0 (2020-04-24) using RStudio ${ }^{47}$, using Seurat $^{48}$ (v3.1.5). We generated visualizations using BioRender (https://biorender.com/) (Fig. 1), dittoSeq ${ }^{49}$ (v1.0.2), a visualization package for analysis and colorblind friendly visualization of single-cell and bulk RNA-Seq data, ggplot2, and UpsetR ${ }^{50}$.

\section{Prefrontal Cortex}

We downloaded a filtered raw expression matrix of 17,296 genes and 70,634 cells from the prefrontal cortex from the AMP-AD Knowledge Portal and used Seurat's Read10x function to generate a count data matrix using the raw count matrix, cell names, and barcodes files provided. A Seurat object was created with the count data matrix and metadata, keeping genes present in at 
least 3 cells, and cells meeting cohort selection criteria with at least 200 genes. Additionally, we selected samples from APOE3/3 and APOE3/4 individuals (Table 1), which resulted in a dataset with 43,831 cells (Supplementary Table 1) and 17,593 genes. Log normalization was performed with a scale.factor of 10,000, and FindVariableFeatures was run using 3,188 features, as specified in the original paper. The data matrix was then scaled with "nCount_RNA" regressed out, and dimensionality reduction was performed with the appropriate dimensions selected based on the corresponding Principal component analysis (PCA) elbow plot. Dimensionality reduction confirmed that there were no batch effects present (Supplementary Fig. 1). As we found the original paper's cell type identification to be comprehensive, we kept the cell type labels for the further analysis (Supplementary Table 1). Due to low cell counts, we did not analyze pericytes and endothelial cells.

\section{Entorhinal Cortex}

A filtered raw expression matrix of 10,850 genes and 13,214 cells from the entorhinal cortex was downloaded from a data repository provided by Grubman et al. Originally composed of 33,694 genes and 14,876 cells, genes and cells were filtered as described by Grubman et al. Cells from $A P O E 3 / 3$ and $A P O E 3 / 4$ individuals were selected (Table 2), and a Seurat object was created to consist of genes in at least 3 cells, and cells with at least 200 genes. Normalization was performed using Seurat's SCTransform method, and Seurat's integration workflow was performed to correct the confounded batches introduced by the experimental design. In this dataset, as shown in Table 2, control samples were processed separately from cases, male samples were processed separately from female samples, and all but one batch contained one APOE genotype. Dimensionality reduction was performed using values from the integrated assay to assess successful batch correction (Supplementary Fig. 5). 
To identify cell types, we adopted techniques from the original paper. Briefly, Grubman et al used Seurat's AddModuleScore function to calculate association scores using lists of brain cell type markers of an unspecified number from the BRETIGEA ${ }^{51}$ package. They labeled cells based on which set of markers they had the highest score for, identified hybrids as cells where the highest and second highest score were within $20 \%$ of each other, and relabeled unidentified cells based on z-score transformation of the gene score distribution. In our case, we used lists of 200 genes for astrocytes, neurons, microglia, oligodendrocytes, OPCs, and endothelial cells to label cells and hybrids to exclude as defined by Grubman et al. We further confirmed successful cell type identification by visualizing scores in a feature plot and assessing homogeneity and separation of clusters in PCA, and Uniform Manifold Approximation and Projection (UMAP) plots based on principal components and expression of top marker genes across cell types. Due to limitations in the number of cells, we excluded endothelial cells from further analyses, which resulted in a dataset comprising 10,850 genes and 9,587 cells (Supplementary Table 2).

\section{Cell type specific $A P O E$-stratified Differential Expression Analysis}

To generate transcriptomic disease signatures relative to $A P O E$ genotype in each cell type, we used Limma-Voom ${ }^{52,53}$.We included sex as a covariate in our design formula, as sex, instead of batch, accounted for the confounding relationships introduced by the original study design, allowed for an appropriate model fit, and avoided the collinearity limitation observed with including batch in the design. Additionally, as samples were age matched, we also did not include age in our design formula. A $d g e$ list object was then created from a matrix of counts extracted from the corresponding Seurat objects. To improve the accuracy of mean-variance 
trend modeling and lower the severity of multiple testing correction, lowly expressed genes were filtered out using edgeR's FilterByExpr with default parameters. Normalization was performed with Trimmed Mean of M-values with singleton pairing (TMMwsp), followed by voom, model fitting with a contrast matrix of each case-control comparison for each cell type-APOE group, and Empirical Bayes fitting of standard errors. We performed a cell type specific AD versus control gene expression comparison in each $A P O E$ variant group separately in our defined prefrontal cortex cohort, entorhinal cohort, and male-only prefrontal cortex cohort, in which we excluded sex as a covariate. Differentially expressed genes were selected using a BenjaminiHochberg (BH) corrected p-value less than 0.05 , and an absolute log base 2-fold change greater than 0.25 .

\section{Functional Enrichment Analysis and Network Visualization}

We performed an overrepresentation analysis of DEGs from the cell type-specific APOEstratified analysis of cells from the prefrontal and entorhinal cortex using gprofiler ${ }^{43}$, a web tool for functional enrichment using an input gene list. We queried differentially expressed genes comparison split by upregulated and downregulated expression to identify enriched pathways. In addition to Gene Ontology cellular components, biological processes, and molecular functions, our enrichment analysis also provided pathways from the Human Protein Atlas, Human Phenotype Ontology, KEGG, Reactome, and Wiki pathways. We followed a previously established protocol ${ }^{54}$ for network enrichment analysis on pathway results derived from our cell type specific DEGs. Briefly, pathway results were imported into the Cytoscape visualization application, EnrichmentMap. We collapsed redundant and related pathways into single biological 
themes and further filtered significant pathways using a BH adjusted p-value $<0.01$. Individual biological themes were defined and summarized using the AutoAnnotate Cytoscape application.

\section{Acknowledgements}

We are grateful to Dr. Martin Kampmann for fruitful discussions and suggestions during the progress of this work. We thank Dr. Katharine Yu, Dr. Idit Kosti, Dr. Dmitry Rychkov, Alice Tang, and the rest of the Sirota lab for scientific guidance and support in this project. This work is funded by the National Institute on Aging (NIA) grants R01AG060393 and R01AG057683. This material is based upon work supported by the National Science Foundation Graduate Research Fellowship Program under Grant No. 1650113. Any opinions, findings, and conclusions or recommendations expressed in this material are those of the author(s) and do not necessarily reflect the views of the National Science Foundation.

\section{Author Contributions}

SB and MS conceived the study. SB designed the study and performed data analysis and interpretation of results (sn-RNAseq differential gene expression analysis and pathway functional enrichment), generated figures, and drafted the manuscript. DB assisted in developing methods and figures for the analysis and drafted the manuscript. AAR assisted in developing methods for data analysis. YL performed network analysis, generated figures for the network analysis, and drafted the manuscript. CWS, TO, AT, BG, KZ, GKF, and YH contributed to the discussion of methods and results as well as the implications of the findings. MS oversaw the study. All authors read and contributed to the final manuscript. The funders had no role in the design, implementation, or preparation of this manuscript. 


\section{Competing Interests}

Y.H. is a cofounder and scientific advisory board member of Escape Bio, Inc., GABAeron, Inc., and Mederon Bio, LLC. M.S. is on the advisory board of twoXAR. Other authors declare no competing financial interests.

\section{References}

1. Lane, C. A., Hardy, J. \& Schott, J. M. Alzheimer's disease. European Journal of Neurology 25, 59-70 (2018).

2. Maresova, P., Hruska, J., Klimova, B., Barakovic, S. \& Krejcar, O. Activities of Daily Living and Associated Costs in the Most Widespread Neurodegenerative Diseases: A Systematic Review. Clin Interv Aging 15, 1841-1862 (2020).

3. Hebert, L. E., Weuve, J., Scherr, P. A. \& Evans, D. A. Alzheimer disease in the United States (2010-2050) estimated using the 2010 census. Neurology 80, 1778-1783 (2013).

4. Long, J. M. \& Holtzman, D. M. Alzheimer Disease: An Update on Pathobiology and Treatment Strategies. Cell 179, 312-339 (2019).

5. Karch, C. M. \& Goate, A. M. Alzheimer's disease risk genes and mechanisms of disease pathogenesis. Biological Psychiatry 77, 43-51 (2015).

6. Yu, J.-T., Tan, L. \& Hardy, J. Apolipoprotein E in Alzheimer's Disease: An Update. Annual Review of Neuroscience 37, 79-100 (2014).

7. Roses, M. D., Allen D. Apolipoprotein e alleles as risk factors in alzheimer's disease. Annu. Rev. Med. 47, 387-400 (1996).

8. Montagne, A. et al. APOE4 leads to blood-brain barrier dysfunction predicting cognitive decline. Nature 1-6 (2020) doi:10.1038/s41586-020-2247-3. 
9. Mahley, R. W., Weisgraber, K. H. \& Huang, Y. Apolipoprotein E4: A causative factor and therapeutic target in neuropathology, including Alzheimer's disease. Proceedings of the National Academy of Sciences of the United States of America 103, 5644-5651 (2006).

10. Wan, Y.-W. et al. Meta-Analysis of the Alzheimer's Disease Human Brain Transcriptome and Functional Dissection in Mouse Models. Cell Rep 32, 107908 (2020).

11. Zhao, N. et al. Apolipoprotein E4 impairs neuronal insulin signaling by trapping insulin receptor in the endosomes. Neuron 96, 115-129.e5 (2017).

12. Zlokovic, B. V. Neurovascular pathways to neurodegeneration in Alzheimer's disease and other disorders. Nature Reviews Neuroscience 12, (2011).

13. Hoe, H.-S., Freeman, J. \& Rebeck, G. W. Apolipoprotein E decreases tau kinases and phospho-tau levels in primary neurons. Molecular Neurodegeneration 1, 18 (2006).

14. Magistri, M., Velmeshev, D., Makhmutova, M. \& Faghihi, M. A. Transcriptomics Profiling of Alzheimer's Disease Reveal Neurovascular Defects, Altered Amyloid- $\beta$ Homeostasis, and Deregulated Expression of Long Noncoding RNAs. J Alzheimers Dis 48, $647-665$.

15. Wang, M. et al. Integrative network analysis of nineteen brain regions identifies molecular signatures and networks underlying selective regional vulnerability to Alzheimer's disease. Genome Medicine 8, 104 (2016).

16. Patel, H., Dobson, R. J. B. \& Newhouse, S. J. A Meta-Analysis of Alzheimer's Disease Brain Transcriptomic Data. Journal of Alzheimer's Disease 68, 1635-1656 (2019).

17. Allen, M. et al. Human whole genome genotype and transcriptome data for Alzheimer's and other neurodegenerative diseases. Scientific Data 3, 1-10 (2016). 
18. Neff, R. A. et al. Molecular subtyping of Alzheimer's disease using RNA sequencing data reveals novel mechanisms and targets. Science Advances 7, eabb5398 (2021).

19. Mathys, H. et al. Single-cell transcriptomic analysis of Alzheimer's disease. Nature 570, 332-337 (2019).

20. Grubman, A. et al. A single-cell atlas of entorhinal cortex from individuals with Alzheimer's disease reveals cell-type-specific gene expression regulation. Nat Neurosci 22, 2087-2097 (2019).

21. Kok, E. H. et al. CLU, CR1 and PICALM genes associate with Alzheimer's-related senile plaques. Alzheimer's Research \& Therapy 3, 12 (2011).

22. Plagman, A. et al. Cholecystokinin and Alzheimer's disease: a biomarker of metabolic function, neural integrity, and cognitive performance. Neurobiology of Aging 76, 201-207 (2019).

23. Mazurek, M. F. \& Beal, F. M. Cholecystokinin and somatostatin in Alzheimer's disease postmortem cerebral cortex. Neurology 41, 716-719 (1991).

24. Chen, X. et al. Cholecystokinin release triggered by NMDA receptors produces LTP and sound-sound associative memory. PNAS 116, 6397-6406 (2019).

25. Jin, Z. et al. Identification and Characterization of Citrulline-modified Brain Proteins by Combining HCD and CID Fragmentation. Proteomics 13, 2682-2691 (2013).

26. Thorsell, A. et al. Neurogranin in cerebrospinal fluid as a marker of synaptic degeneration in Alzheimer's disease. Brain Research 1362, 13-22 (2010).

27. Philip, D. et al. Dihydrofolate reductase 19-bp deletion polymorphism modifies the association of folate status with memory in a cross-sectional multi-ethnic study of adults 123. Am J Clin Nutr 102, 1279-1288 (2015). 
28. Cario, H. et al. Dihydrofolate Reductase Deficiency Due to a Homozygous DHFR Mutation Causes Megaloblastic Anemia and Cerebral Folate Deficiency Leading to Severe Neurologic Disease. The American Journal of Human Genetics 88, 226-231 (2011).

29. Mouton-Liger, F. et al. CSF levels of the BACE1 substrate NRG1 correlate with cognition in Alzheimer's disease. Alzheimer's Research \& Therapy 12, 88 (2020).

30. Mitchell, R. M. et al. ErbB4 reduces synaptic GABAA currents independent of its receptor tyrosine kinase activity. Proc Natl Acad Sci U S A 110, 19603-19608 (2013).

31. Mozhui, K. et al. Genetic regulation of Nrnx1 expression: an integrative cross-species analysis of schizophrenia candidate genes. Transl Psychiatry 1, e25 (2011).

32. Nishikimi, A., Kukimoto-Niino, M., Yokoyama, S. \& Fukui, Y. Immune regulatory functions of DOCK family proteins in health and disease. Experimental Cell Research 319, 2343-2349 (2013).

33. Mahajan, G. J. et al. Altered neuro-inflammatory gene expression in hippocampus in major depressive disorder. Progress in Neuro-Psychopharmacology and Biological Psychiatry 82, 177-186 (2018).

34. Bik-Multanowski, M., Pietrzyk, J. J. \& Midro, A. MTRNR2L12: A Candidate Blood Marker of Early Alzheimer's Disease-Like Dementia in Adults with Down Syndrome. J Alzheimers Dis 46, 145-150 (2015).

35. Mahmud, F. J. et al. Osteopontin/secreted phosphoprotein-1 behaves as a molecular brake regulating the neuroinflammatory response to chronic viral infection. $J$ Neuroinflammation 17, 273 (2020). 
36. Wen, H. et al. Neuroglobin mediates neuroprotection of hypoxic postconditioning against transient global cerebral ischemia in rats through preserving the activity of $\mathrm{Na}+/ \mathrm{K}+$ ATPases. Cell Death \& Disease 9, 1-18 (2018).

37. Hua, Y., Zhao, H., Lu, X., Kong, Y. \& Jin, H. Meta-Analysis of the Cystatin C(CST3) Gene G73A Polymorphism and Susceptibility to Alzheimer's Disease. International Journal of Neuroscience 122, 431-438 (2012).

38. Shin, J.-G. et al. Putative association of GPC5 polymorphism with the risk of inflammatory demyelinating diseases. Journal of the Neurological Sciences 335, 82-88 (2013).

39. Zhou, Y., Zhang, X. \& Klibanski, A. MEG3 noncoding RNA: a tumor suppressor. J Mol Endocrinol 48, R45-53 (2012).

40. Koenning, M. et al. Myelin gene regulatory factor is required for maintenance of myelin and mature oligodendrocyte identity in the adult CNS. J Neurosci 32, 12528-12542 (2012).

41. Ferrer, I. \& Andrés-Benito, P. White matter alterations in Alzheimer's disease without concomitant pathologies. Neuropathol Appl Neurobiol 46, 654-672 (2020).

42. Ousman, S. S. et al. Protective and therapeutic role for $\alpha \mathrm{B}$-crystallin in autoimmune demyelination. Nature 448, 474-479 (2007).

43. Raudvere, U. et al. g:Profiler: a web server for functional enrichment analysis and conversions of gene lists (2019 update). Nucleic Acids Res 47, W191-W198 (2019).

44. Satoh, J., Tabunoki, H., Yamamura, T., Arima, K. \& Konno, H. TROY and LINGO-1 expression in astrocytes and macrophages/microglia in multiple sclerosis lesions. Neuropathol Appl Neurobiol 33, 99-107 (2007). 
45. Khan, U. A. et al. Molecular drivers and cortical spread of lateral entorhinal cortex dysfunction in preclinical Alzheimer's disease. Nature Neuroscience 17, 304-311 (2014).

46. R Core Team. R: A Language and Environment for Statistical Computing. https://www.rproject.org/ (2020).

47. R Studio Team. RStudio: Integrated Development Environment for R. https://rstudio.com/ (2020).

48. Stuart, T. et al. Comprehensive Integration of Single-Cell Data. Cell 177, 1888-1902.e21 (2019).

49. Bunis, D. G., Andrews, J., Fragiadakis, G. K., Burt, T. D. \& Sirota, M. dittoSeq: universal user-friendly single-cell and bulk RNA sequencing visualization toolkit. Bioinformatics (2020) doi:10.1093/bioinformatics/btaa1011.

50. Conway, J. R., Lex, A. \& Gehlenborg, N. UpSetR: an R package for the visualization of intersecting sets and their properties. Bioinformatics 33, 2938-2940 (2017).

51. McKenzie, A. T. et al. Brain Cell Type Specific Gene Expression and Co-expression Network Architectures. Scientific Reports 8, 1-19 (2018).

52. Ritchie, M. E. et al. limma powers differential expression analyses for RNA-sequencing and microarray studies. Nucleic Acids Res 43, e47 (2015).

53. Phipson, B., Lee, S., Majewski, I. J., Alexander, W. S. \& Smyth, G. K. Robust hyperparameter estimation protects against hypervariable genes and improves power to detect differential expression. Ann Appl Stat 10, 946-963 (2016).

54. Reimand, J. et al. Pathway enrichment analysis and visualization of omics data using g:Profiler, GSEA, Cytoscape and EnrichmentMap. Nature Protocols 14, 482-517 (2019). 


\section{Figure legends}

Figure 1: Overview of cohort sample definition and workflow for $A P O E$ genotype stratified cell type specific differential gene expression and functional enrichment. AD and non-AD cells were determined based on tau tangle (Braak) and amyloid $\beta$ plaque (CERAD) burden. Cell types were identified, and AD versus non-AD differential expression and pathway network enrichment analyses were performed separately for APOE3/3 and APOE3/4 cells of each cell type.

Figure 2: $A P O E$ genotype stratified cell type specific disease signatures in the prefrontal cortex. a. AD versus non-AD differentially expressed gene (DEG) counts for astrocytes (Ast), excitatory (Ex) and inhibitory (In) neurons, microglia (Mic), oligodendrocytes (Oli), and oligodendrocyte progenitor cells (Opc) in surveyed APOE genotypes. DEGs were selected using a Benjamini Hochberg adjusted p-value $<0.05$ and absolute $\log 2$ fold change $(\mathrm{FC})>0.25, \mathrm{~b}$. Subset of DEGs shared by both $A P O E$ genotypes and their corresponding log2 FC, c. Pairwise DEG plots of DEGs in APOE3/3 and APOE3/4 samples using $\log 2 \mathrm{FC}$ scores. Genes shown are significant and have a $\log 2 \mathrm{FC}>0.25$ in at least one $A P O E$ genotype. Colors indicate significance level of DEGs and whether DEGs are unique or shared by $A P O E$ genotypes, d. $\log 2$ $\mathrm{FC}$ scores of all genes in the DE analysis clustered by cell type and APOE genotype.

Figure 3: Shared and unique disease signatures across cell types in APOE3/3 and APOE3/4 prefrontal cortex samples. a. Upset plots indicating intersections of $A D$ versus non-AD differentially expressed genes (DEGs) (Benjamini Hochberg (BH) adjusted p-value $<0.05$ and absolute $\log _{2}$ fold change $\left.(\mathrm{FC})>0.25\right)$ across cell types. Rows correspond to cell types. The bar chart shows the number of single and common sets of DEGs across cell types. Single filled dots represent a unique set of DEGs for the corresponding cell type. Multiple filled black dots connected by vertical lines represent common sets of DEGs across cell types, b. LINGO1, $R A S G E F 1 B, N R X N 1$ and $C L U$ expression. Asterisks represent meeting both significance (BH adjusted p-value $<0.05)$, and absolute $\log _{2} \mathrm{FC}(>0.25)$ thresholds. Colors correspond to $A P O E$ genotype and $\mathrm{AD}$ status.

Figure 4: $A P O E$ genotype stratified cell type specific disease signatures in the entorhinal cortex. a. AD versus non-AD differentially expressed gene (DEG) counts for astrocytes (Ast, neurons (Neu), microglia (Mic), oligodendrocytes (Oli), and oligodendrocyte progenitor cells $(\mathrm{Opc})$ in surveyed APOE genotypes. DEGs were selected using a Benjamini Hochberg adjusted p-value $<0.05$ and absolute $\log 2$ fold change $(F C)>0.25$, b. Pairwise DEG plots of DEGs in APOE3/3 and APOE3/4 samples using $\log 2$ FC scores. Genes shown are significant and have a $\log 2 \mathrm{FC}>0.25$ in at least one $A P O E$ genotype. Colors indicate significance level of DEGs and whether DEGs are unique or shared by APOE genotypes, c. $\log 2 \mathrm{FC}$ scores of all genes in the $\mathrm{DE}$ analysis clustered by cell type and $A P O E$ genotype

Figure 5: $A P O E$ genotype stratified cell type specific disease signatures across brain regions. a. Upset plots indicating intersections of $\mathrm{AD}$ versus non-AD differentially expressed genes (DEGs) (Benjamini Hochberg $(\mathrm{BH})$ adjusted p-value $<0.05$ and absolute log2 fold change $(\mathrm{FC})>0.25$ ) within cell types across brain region and $A P O E$ genotype. Rows correspond to brain region and $A P O E$ genotype pairings. The bar chart shows the number of single and common sets 
of DEGs across brain regions and $A P O E$ genotype pairings. Single filled dots represent a unique set of DEGs for the corresponding brain region and $A P O E$ genotype pairing. Multiple filled black dots connected by vertical lines represent common sets of DEGs across brain region and APOE genotype pairings. Bar chart colors correspond to whether DEGs are shared between brain regions or APOE genotype using the bottom right key, b. $\log 2 \mathrm{FC}$ scores of all genes in the DE analysis of both brain regions clustered by cell type, brain region, and $A P O E$ genotype.

Figure 6: Enriched disease pathway networks in $A P O E 3 / 3$ and $A P O E 3 / 4$ cells. AD compared to non-AD functionally enriched pathways with a (Benjamini Hochberg $(\mathrm{BH})$ adjusted p-value $<0.01$ clustered into biological themes for: a. astrocytes of the prefrontal cortex, $b$. microglia of the entorhinal cortex, and c. prefrontal cortex excitatory (Ex) and inhibitory (In) neurons, and entorhinal cortex undistinguished neurons (Neu). Lines represent gene set overlaps with magnitude showed by thickness.

Supplementary Figure 1: Dimensionality reduction of prefrontal and entorhinal cortices cohort cells by $A P O E$ genotype, batch, cell type, diagnosis, and sex. a. Uniform Manifold Approximation and Projection (UMAP) of prefrontal cortex cohort, where each point represents a cell, b. UMAP of entorhinal cortex cohort before batch correction, c. UMAP of entorhinal cortex cohort after batch correction.

Supplementary Figure 2: Shared and unique disease signatures within cell types across APOE3/3 and APOE3/4 samples in the prefrontal and entorhinal cortices. a. Upset plots indicating intersections of $\mathrm{AD}$ versus non-AD differentially expressed genes (DEGs) (Benjamini Hochberg $(\mathrm{BH})$ adjusted p-value $<0.05$ and absolute $\log _{2}$ fold change $\left.(\mathrm{FC})>0.25\right)$ across $A P O E$ genotypes. Rows correspond to cell type and $A P O E$ genotype pairings. The bar chart shows the number of single and common sets of DEGs across $A P O E$ genotypes. Single filled dots represent a unique set of DEGs for the corresponding $A P O E$ genotype. Multiple filled black dots connected by vertical lines represent common sets of DEGs across APOE genotypes.

Supplementary Figure 3: $A P O E$ genotype stratified cell type specific disease signatures in male prefrontal cortex. a. AD versus non-AD differentially expressed gene (DEG) counts for astrocytes (Ast), excitatory (Ex) and inhibitory (In) neurons, microglia (Mic), oligodendrocytes (Oli), and oligodendrocyte progenitor cells (Opc) in surveyed $A P O E$ genotypes. DEGs were selected using a Benjamini Hochberg adjusted p-value $<0.05$ and absolute log2 fold change (FC) $>0.25, \mathrm{~b} . \log 2 \mathrm{FC}$ scores of all genes in the DE analysis clustered by cell type and APOE genotype, c. Pairwise DEG plots of DEGs in APOE3/3 and APOE3/4 samples using log2 FC scores. Genes shown are significant and have a $\log 2 \mathrm{FC}>0.25$ in at least one APOE genotype. Colors indicate significance level of DEGs and whether DEGs are unique or shared by $A P O E$ genotypes.

Supplementary Figure 4 Shared and unique disease signatures across cell types in APOE3/3 and APOE3/4 entorhinal cortex samples. a. Upset plots indicating intersections of $\mathrm{AD}$ versus non-AD differentially expressed genes (DEGs) (Benjamini Hochberg (BH) adjusted p-value $<0.05$ and absolute $\log 2$ fold change $(\mathrm{FC})>0.25)$ across cell types. Rows correspond to cell types. The bar chart shows the number of single and common sets of DEGs across cell types. Single filled dots represent a unique set of DEGs for the corresponding cell type. Multiple filled 
black dots connected by vertical lines represent common sets of DEGs across cell types, b. LINGO1, FTL, NRXN1 and ADGRL3 expression. Asterisks represent meeting both significance (BH adjusted p-value $<0.05)$, and absolute $\log _{2} \mathrm{FC}(>0.25)$ thresholds. Colors correspond to $A P O E$ genotype and AD status.

Supplementary Figure 5: Enriched disease pathway networks in $A P O E 3 / 3$ and $A P O E 3 / 4$ prefrontal cortex cells. AD compared to non-AD functionally enriched pathways with a (Benjamini Hochberg $(\mathrm{BH})$ adjusted p-value $<0.01$ clustered into biological themes for excitatory and inhibitory neurons, microglia, oligodendrocytes, and oligodendrocyte progenitor cells. Lines represent gene set overlaps with magnitude showed by thickness.

\section{Supplementary Figure 6: Enriched disease pathway networks in $A P O E 3 / 3$ and $A P O E 3 / 4$} entorhinal cortex cells. AD compared to non-AD functionally enriched pathways with a (Benjamini Hochberg $(\mathrm{BH})$ adjusted p-value $<0.01$ clustered into biological themes for neurons, oligodendrocyte progenitor cells, astrocytes, and oligodendrocytes. Lines represent gene set overlaps with magnitude showed by thickness. 
Tables

Table 1: Prefrontal cortex cohort

\begin{tabular}{|c|c|c|c|c|c|}
\hline ID & Sex & APOE & Age & Diagnosis & Batch \\
\hline $\mathrm{AD} 1$ & Female & $3 / 3$ & 90 & $\mathrm{AD}$ & 3 \\
\hline $\mathrm{AD} 2$ & Female & $3 / 4$ & 90 & $\mathrm{AD}$ & 11 \\
\hline AD3 & Female & $3 / 3$ & 90 & $\mathrm{AD}$ & 5 \\
\hline $\mathrm{AD} 4$ & Female & $3 / 3$ & 90 & $\mathrm{AD}$ & 8 \\
\hline AD5 & Female & $3 / 3$ & 87 & $\mathrm{AD}$ & 10 \\
\hline AD6 & Female & $3 / 4$ & 76 & $\mathrm{AD}$ & 3 \\
\hline $\mathrm{AD} 7$ & Female & $3 / 4$ & 74 & $\mathrm{AD}$ & 2 \\
\hline AD8 & Male & $3 / 3$ & 89 & $\mathrm{AD}$ & 5 \\
\hline AD9 & Male & $3 / 4$ & 89 & $\mathrm{AD}$ & 1 \\
\hline $\mathrm{AD} 10$ & Male & $3 / 3$ & 87 & $\mathrm{AD}$ & 10 \\
\hline AD11 & Male & $3 / 4$ & 85 & $\mathrm{AD}$ & 4 \\
\hline $\mathrm{AD} 12$ & Male & $3 / 4$ & 86 & $\mathrm{AD}$ & 9 \\
\hline $\mathrm{AD} 13$ & Male & $3 / 3$ & 83 & $\mathrm{AD}$ & 4 \\
\hline $\mathrm{AD} 14$ & Male & $3 / 3$ & 86 & $\mathrm{AD}$ & 2 \\
\hline AD15 & Male & $3 / 3$ & 80 & $\mathrm{AD}$ & 8 \\
\hline $\mathrm{Cl}$ & Female & $3 / 3$ & 90 & Control & 11 \\
\hline $\mathrm{C} 2$ & Female & $3 / 3$ & 87 & Control & 9 \\
\hline $\mathrm{C} 3$ & Female & $3 / 3$ & 87 & Control & 10 \\
\hline $\mathrm{C} 4$ & Female & $3 / 3$ & 83 & Control & 6 \\
\hline C5 & Female & $3 / 3$ & 81 & Control & 7 \\
\hline $\mathrm{C} 6$ & Female & $3 / 3$ & 79 & Control & 3 \\
\hline $\mathrm{C} 7$ & Male & $3 / 3$ & 90 & Control & 5 \\
\hline $\mathrm{C} 8$ & Male & $3 / 4$ & 88 & Control & 1 \\
\hline $\mathrm{C} 9$ & Male & $3 / 3$ & 87 & Control & 12 \\
\hline $\mathrm{C} 10$ & Male & $3 / 3$ & 84 & Control & 4 \\
\hline Cl1 & Male & $3 / 3$ & 80 & Control & 8 \\
\hline $\mathrm{C} 12$ & Male & $3 / 3$ & 80 & Control & 1 \\
\hline $\mathrm{C} 13$ & Male & $3 / 3$ & 80 & Control & 9 \\
\hline $\mathrm{Cl} 4$ & Male & $3 / 3$ & 79 & Control & 2 \\
\hline C15 & Male & $3 / 3$ & 76 & Control & 4 \\
\hline
\end{tabular}

Table 2: Entorhinal cortex cohort

\begin{tabular}{|c|c|c|c|c|c|}
\hline ID & Sex & APOE & Age & Diagnosis & Batch \\
\hline AD1 & Male & $3 / 4$ & 91 & AD & AD1_AD2 \\
\hline AD2 & Male & $3 / 4$ & 83.8 & AD & AD1_AD2 \\
\hline AD3 & Female & $3 / 3$ & 83.0 & AD & AD3_AD4 \\
\hline AD4 & Male & $3 / 4$ & 74.6 & AD & AD5_AD6 \\
\hline C1 & Female & $3 / 3$ & 67.3 & Control & Ct1_Ct2 \\
\hline C2 & Female & $3 / 3$ & 82.7 & Control & Ct1_Ct2 \\
\hline C3 & Male & $3 / 3$ & 72.6 & Control & Ct3_Ct4 \\
\hline C4 & Male & $3 / 4$ & 75.6 & Control & Ct3_Ct4 \\
\hline C5 & Male & $3 / 3$ & 77.5 & Control & Ct5_Ct6 \\
\hline \hline
\end{tabular}



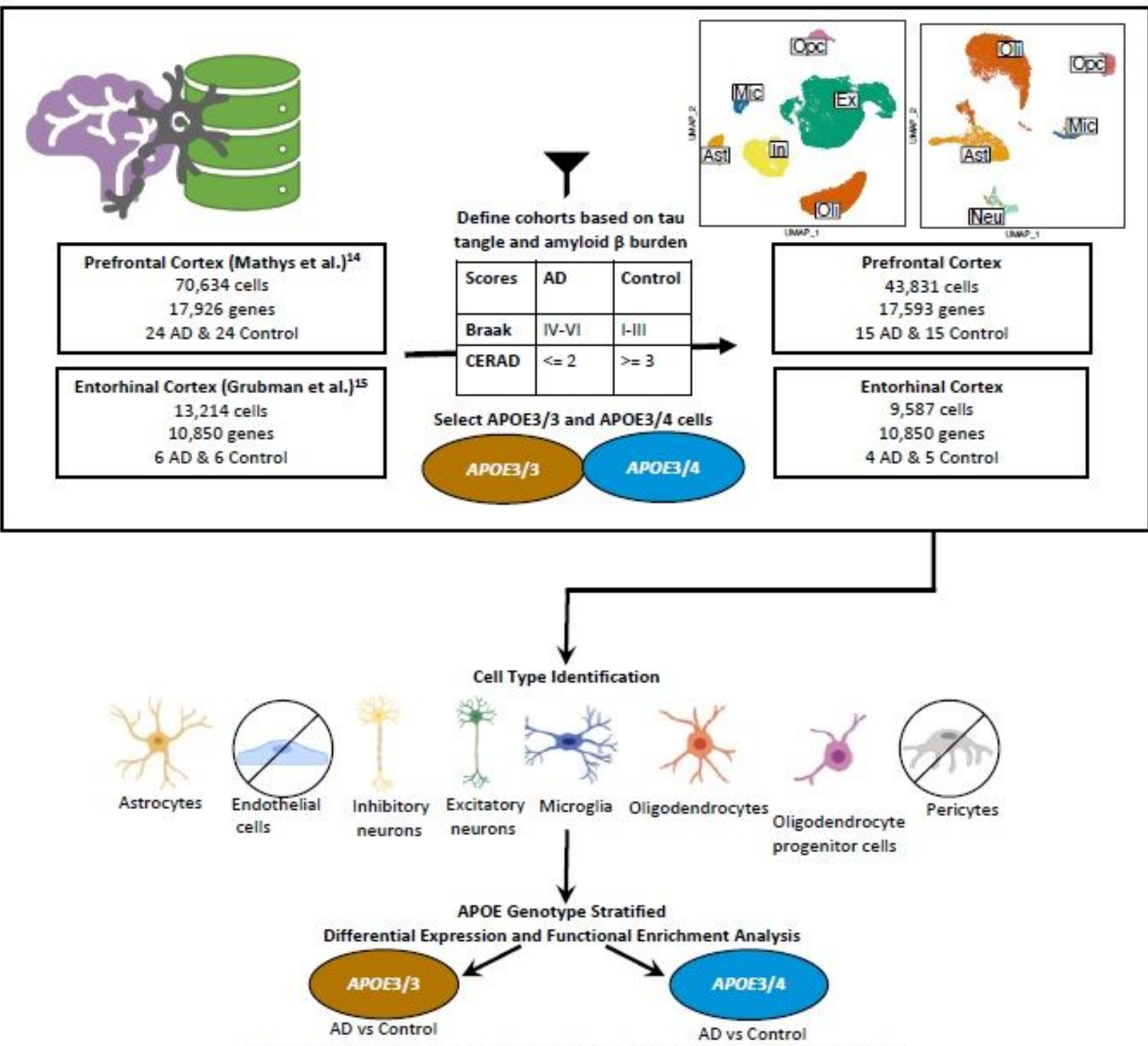

[Benjamini Hochberg adjusted $p$ value $<0.05$ and absolute $\log 2$ fold change $>0.25$ ]
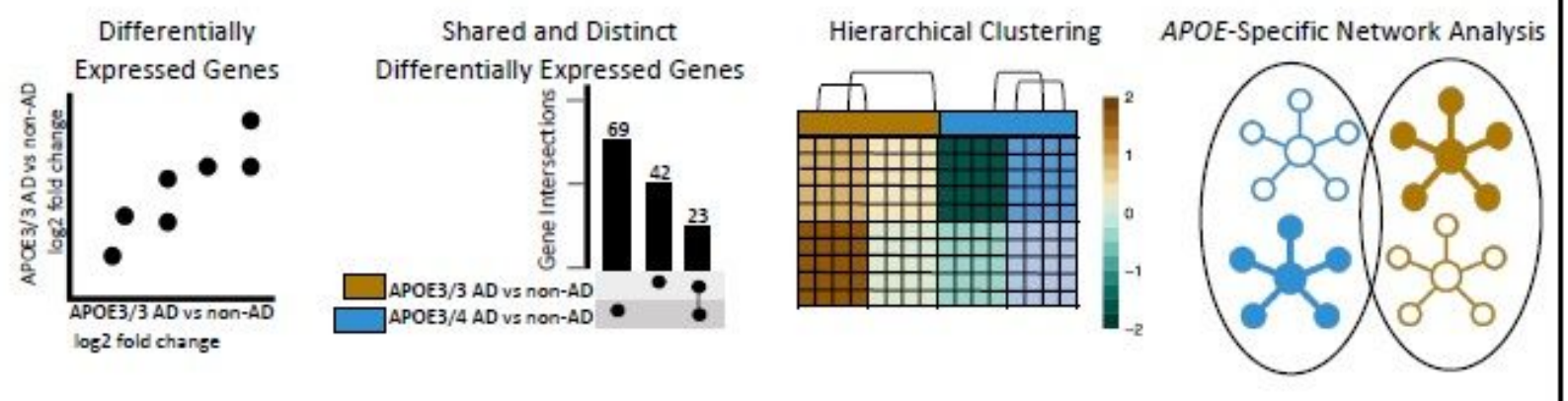

Figure 1

Overview of cohort sample definition and workflow for APOE genotype stratified cell type specific differential gene expression and functional enrichment. $A D$ and non-AD cells were determined based on tau tangle (Braak) and amyloid $\beta$ plaque (CERAD) burden. Cell types were identified, and AD versus non- 
$A D$ differential expression and pathway network enrichment analyses were performed separately for APOE3/3 and APOE3/4 cells of each cell type.

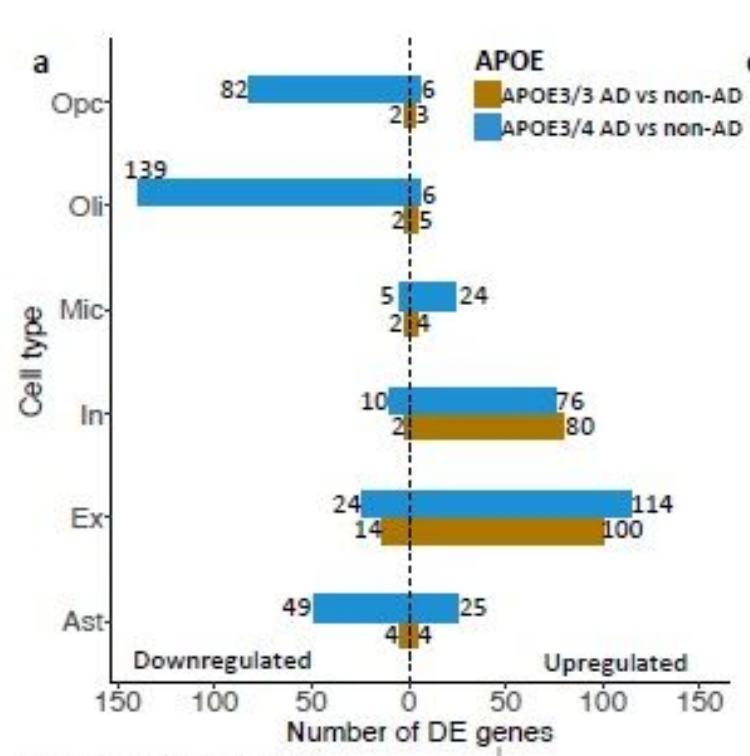

\section{Adjusted p-value $(\mathrm{BH})$}

- $<0.05$ in APOE $3 / 3$ AD vs non-AD DEGs

$<0.05$ in APOE3/4 AD vs non-AD DEGs

- $<0.05$ in Both APOE genotypes \& Opposite Direction

$<0.05$ in Both APOE genotypes \& Same Direction

- Not Significant

\begin{tabular}{|l|l|l|l|}
\hline Gene & $\begin{array}{c}\text { Cell } \\
\text { type }\end{array}$ & $\begin{array}{c}\text { APOE3/3 } \\
\text { Log2 FC }\end{array}$ & $\begin{array}{c}\text { APOE3/4 } \\
\text { Log2 FC }\end{array}$ \\
\hline bCK & Ex & -0.228 & -0.664 \\
\hline CLU & Ast & 0.268 & 1.032 \\
\hline & Ex & 0.234 & 0.368 \\
\hline NRGN & In & 0.255 & 0.578 \\
\hline & Ex & -0.036 & -0.608 \\
\hline In & 0.309 & 0.460 \\
\hline OHFR & Oli & -0.086 & -0.295 \\
\hline & Ex & 1.063 & 0.415 \\
\hline & In & 0.611 & 0.418 \\
\hline Oli & -0.065 & -0.473 \\
\hline ERBB4 & Ex & 0.466 & 0.627 \\
\hline Ast & -0.269 & -0.305 \\
\hline & Oli & -0.091 & -0.265 \\
\hline
\end{tabular}
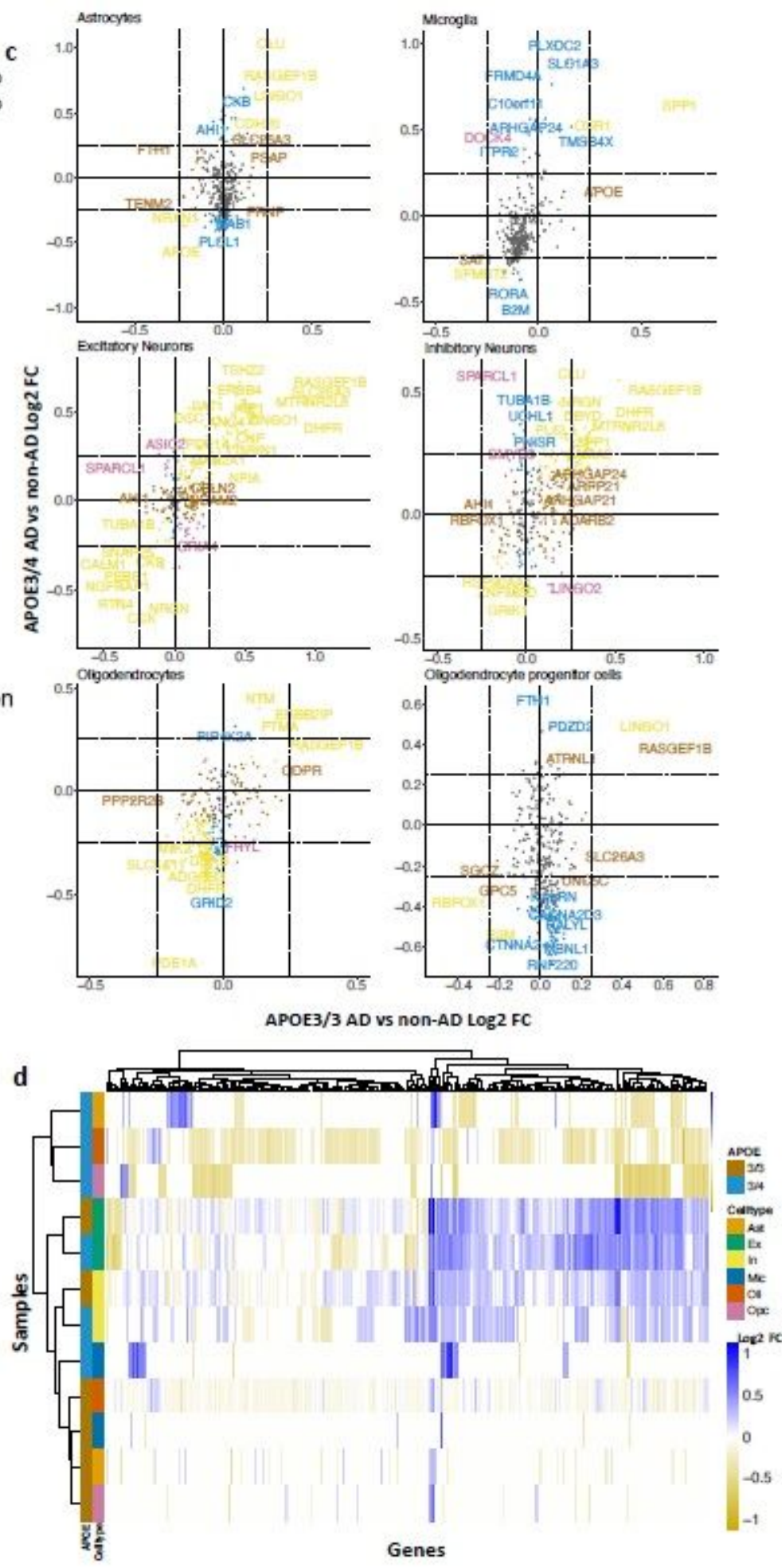

Figure 2

APOE genotype stratified cell type specific disease signatures in the prefrontal cortex. a. AD versus nonAD differentially expressed gene (DEG) counts for astrocytes (Ast), excitatory (Ex) and inhibitory (In) neurons, microglia (Mic), oligodendrocytes (Oli), and oligodendrocyte progenitor cells (Opc) in surveyed 
APOE genotypes. DEGs were selected using a Benjamini Hochberg adjusted $p$-value $<0.05$ and absolute log2 fold change $(F C)>0.25$, b. Subset of DEGs shared by both APOE genotypes and their corresponding $\log 2 \mathrm{FC}$, c. Pairwise DEG plots of DEGs in APOE3/3 and APOE3/4 samples using log2 FC scores. Genes shown are significant and have a log2 $F C>0.25$ in at least one APOE genotype. Colors indicate significance level of DEGs and whether DEGs are unique or shared by APOE genotypes, $d$. log2 FC scores of all genes in the DE analysis clustered by cell type and APOE genotype.

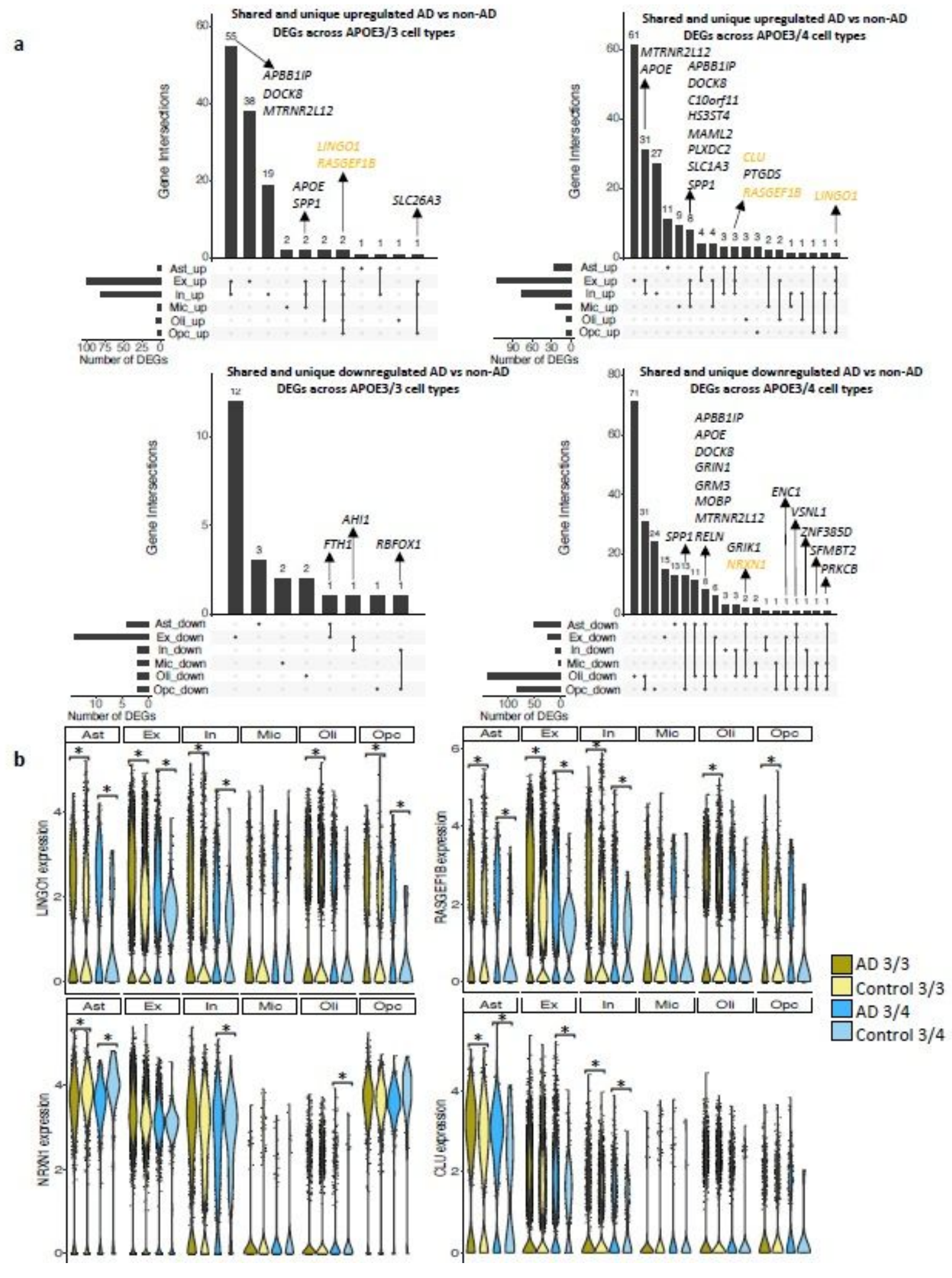

Figure 3 
Shared and unique disease signatures across cell types in APOE3/3 and APOE3/4 prefrontal cortex samples. a. Upset plots indicating intersections of $A D$ versus non- $A D$ differentially expressed genes (DEGs) (Benjamini Hochberg (BH) adjusted $p$-value $<0.05$ and absolute log2 fold change $(F C)>0.25$ ) across cell types. Rows correspond to cell types. The bar chart shows the number of single and common sets of DEGs across cell types. Single filled dots represent a unique set of DEGs for the corresponding cell type. Multiple filled black dots connected by vertical lines represent common sets of DEGs across cell types, b. LING01, RASGEF1B, NRXN1 and CLU expression. Asterisks represent meeting both significance (BH adjusted p-value < 0.05), and absolute log2 FC $(>0.25)$ thresholds. Colors correspond to APOE genotype and $A D$ status. 

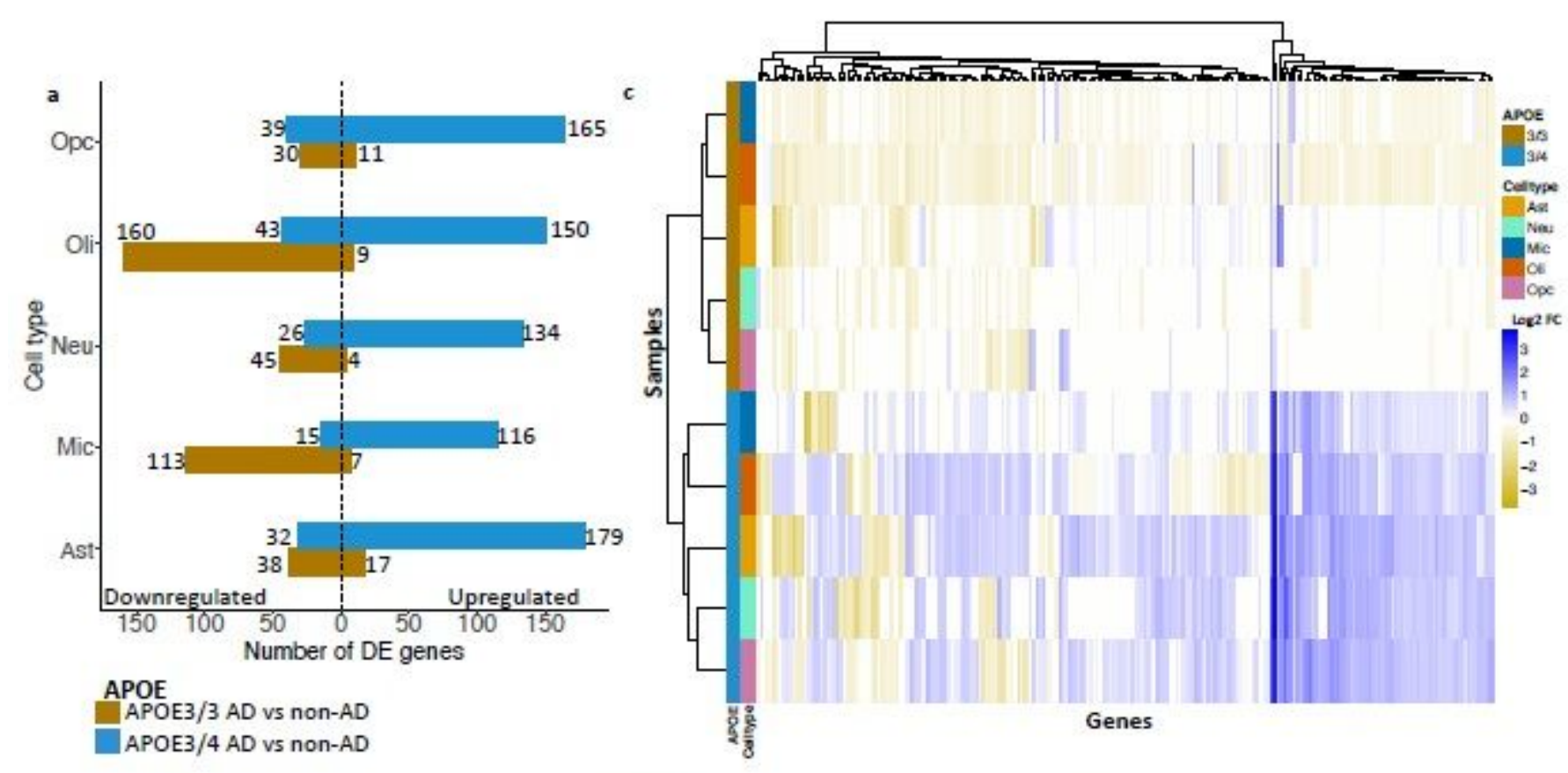

b
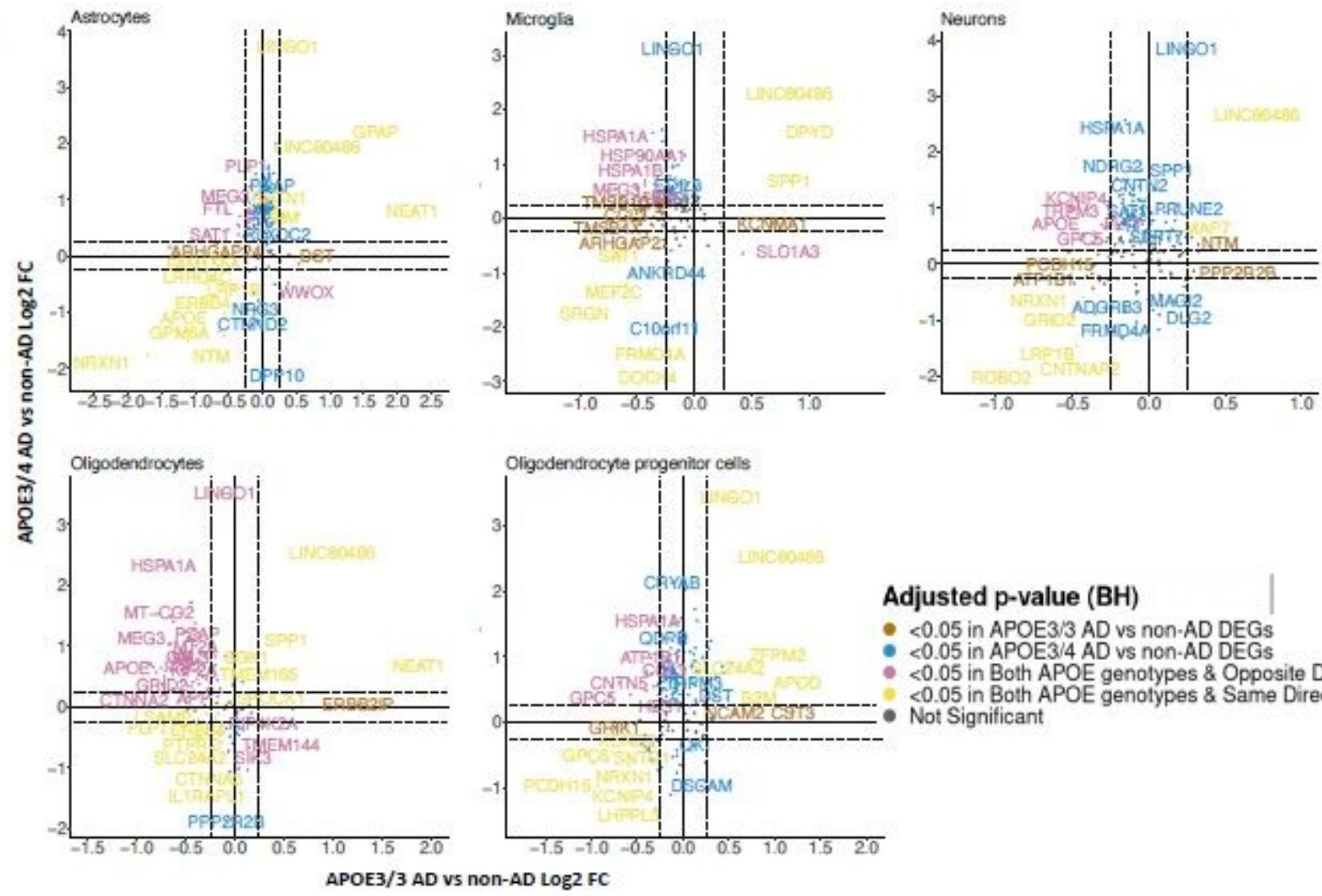

\section{Adjusted p-value (BH)}

$<0.05$ in APOE $3 / 3$ AD vs non-AD DEGs

$<0.05$ in APOE3/4 AD vs non-AD DEGs

$<0.05$ in Both APOE genotypes \& Opposite Direction $<0.05$ in Both APOE genotypes \& Same Direction Not Significant

\section{Figure 4}

APOE genotype stratified cell type specific disease signatures in the entorhinal cortex. a. AD versus nonAD differentially expressed gene (DEG) counts for astrocytes (Ast, neurons (Neu), microglia (Mic), oligodendrocytes (Oli), and oligodendrocyte progenitor cells (Opc) in surveyed APOE genotypes. DEGs were selected using a Benjamini Hochberg adjusted $p$-value $<0.05$ and absolute log 2 fold change (FC) > 0.25 , b. Pairwise DEG plots of DEGs in APOE3/3 and APOE3/4 samples using log2 FC scores. Genes 
shown are significant and have a log2 FC $>0.25$ in at least one APOE genotype. Colors indicate significance level of DEGs and whether DEGs are unique or shared by APOE genotypes, c. log2 FC scores of all genes in the DE analysis clustered by cell type and APOE genotype

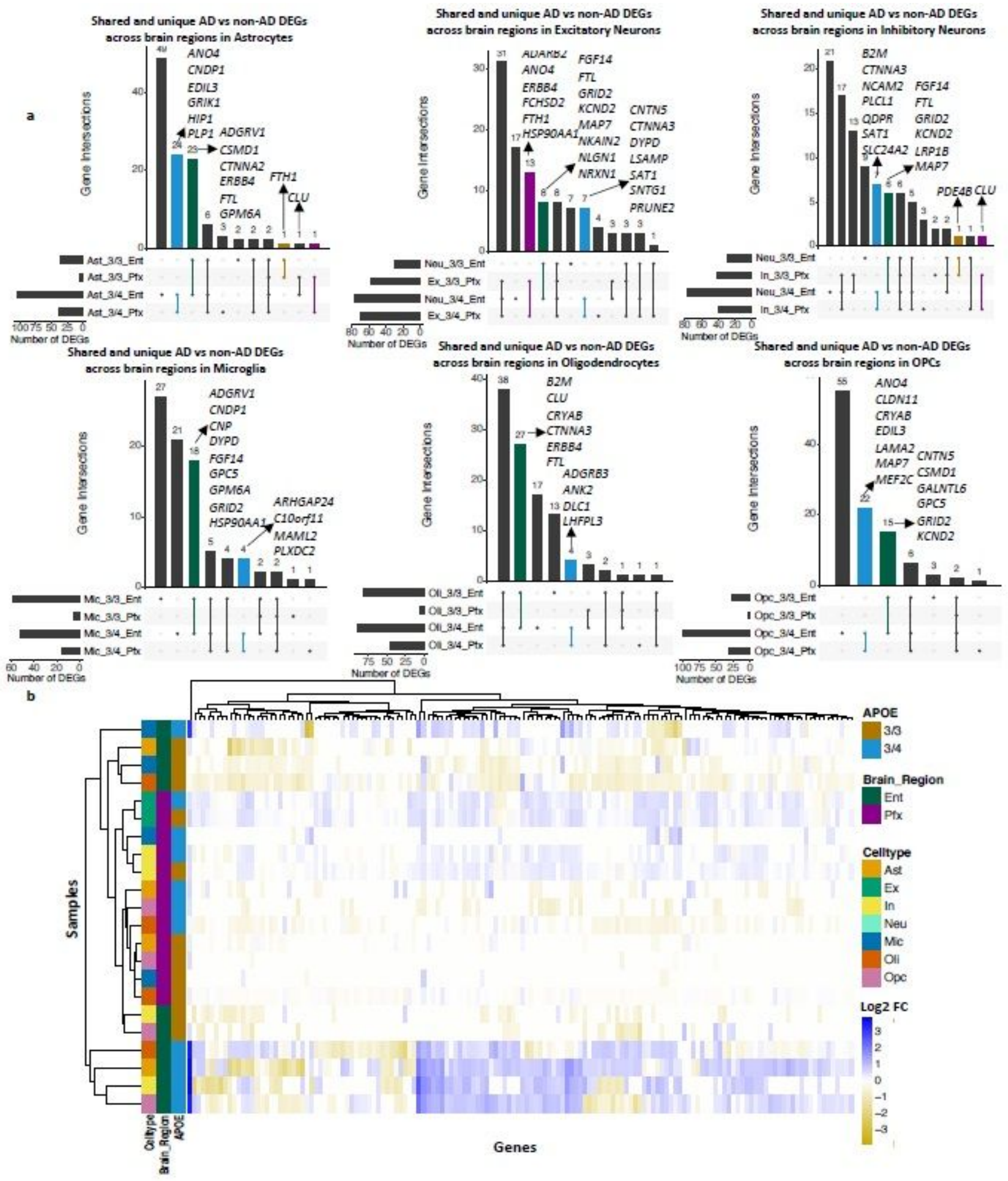

Figure 5

APOE genotype stratified cell type specific disease signatures across brain regions. a. Upset plots indicating intersections of $A D$ versus non-AD differentially expressed genes (DEGs) (Benjamini Hochberg 
$(\mathrm{BH})$ adjusted $p$-value $<0.05$ and absolute log2 fold change $(\mathrm{FC})>0.25)$ within cell types across brain region and APOE genotype. Rows correspond to brain region and APOE genotype pairings. The bar chart shows the number of single and common sets of DEGs across brain regions and APOE genotype pairings. Single filled dots represent a unique set of DEGs for the corresponding brain region and APOE genotype pairing. Multiple filled black dots connected by vertical lines represent common sets of DEGs across brain region and APOE genotype pairings. Bar chart colors correspond to whether DEGs are shared between brain regions or APOE genotype using the bottom right key, b. log2 FC scores of all genes in the $\mathrm{DE}$ analysis of both brain regions clustered by cell type, brain region, and APOE genotype. 

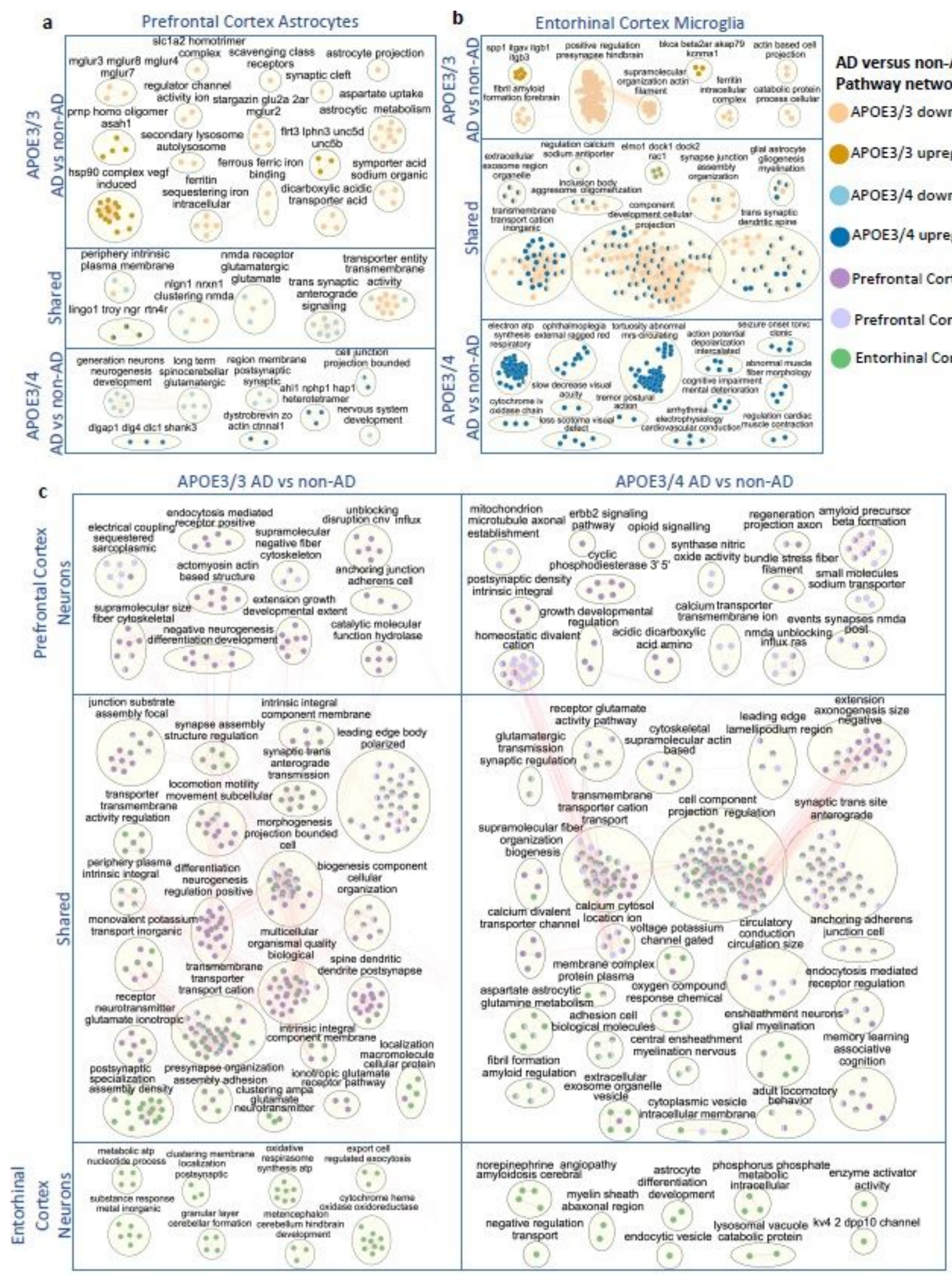

\section{Figure 6}

Enriched disease pathway networks in APOE3/3 and APOE3/4 cells. AD compared to non-AD functionally enriched pathways with a (Benjamini Hochberg $(\mathrm{BH})$ adjusted p-value $<0.01$ clustered into biological themes for: a. astrocytes of the prefrontal cortex, b. microglia of the entorhinal cortex, and c. prefrontal cortex excitatory (Ex) and inhibitory (In) neurons, and entorhinal cortex undistinguished neurons (Neu). Lines represent gene set overlaps with magnitude showed by thickness.

\section{$A D$ versus non- $A D$ \\ Pathway networks \\ APOE $3 / 3$ downregulated \\ APOE $3 / 3$ upregulated \\ APOE $3 / 4$ downregulated \\ APOE3/4 upregulated \\ Prefrontal Cortex Ex \\ Prefrontal Cortex In \\ Entorhinal Cortex Neu}

APOE3/4 AD vs non-AD 


\section{Supplementary Files}

This is a list of supplementary files associated with this preprint. Click to download.

- SupplementaryTable1.txt

- SupplementaryTable2.txt

- SupplementaryTable3.txt

- SupplementaryTable4.txt

- SupplementaryTable5.txt

- SupplementaryTables69.xIsx

- SupplementalFigures.pdf 